\title{
Multimodality of rich clusters from the SDSS DR8 within the supercluster-void network ${ }^{\star}$
}

\author{
M. Einasto ${ }^{1}$, L. J. Liivamägi ${ }^{1,2}$, E. Tempel ${ }^{1,3}$, E. Saar ${ }^{1,4}$, J. Vennik ${ }^{1}$, P. Nurmi ${ }^{5}$, M. Gramann ${ }^{1}$, J. Einasto ${ }^{1,4,6}$, E. Tago ${ }^{1}$, \\ P. Heinämäki ${ }^{5}$, A. Ahvensalmi ${ }^{5}$, and V. J. Martínez ${ }^{7}$
}

1 Tartu Observatory, 61602 Tõravere, Estonia e-mail: maret@aai.ee

${ }^{2}$ Institute of Physics, Tartu University, Tähe 4, 51010 Tartu, Estonia

3 National Institute of Chemical Physics and Biophysics, Tallinn 10143, Estonia

${ }^{4}$ Estonian Academy of Sciences, 10130 Tallinn, Estonia

5 Tuorla Observatory, University of Turku, Väisäläntie 20, Piikkiö, Finland

${ }^{6}$ ICRANet, Piazza della Repubblica 10, 65122 Pescara, Italy

7 Observatori Astronòmic, Universitat de València, Apartat de Correus 22085, 46071 València, Spain

Received 27 February 2012 / Accepted 4 April 2012

\begin{abstract}
Context. The study of the properties of galaxy clusters and their environment gives us information about the formation and evolution of galaxies, groups and clusters, and larger structures - superclusters of galaxies and the whole cosmic web.

Aims. We study the relations between the multimodality of galaxy clusters drawn from the SDSS DR8 and the environment where they reside. As cluster environment we consider the global luminosity density field, supercluster membership, and supercluster morphology.

Methods. We use 3D normal mixture modelling, the Dressler-Shectman test, and the peculiar velocity of cluster main galaxies as signatures of multimodality of clusters. We calculate the luminosity density field to study the environmental densities around clusters, and to find superclusters where clusters reside. We determine the morphology of superclusters with the Minkowski functionals and compare the properties of clusters in superclusters of different morphology. We apply principal component analysis to study the relations between the multimodality parameters of clusters and their environment simultaneously.

Results. Multimodal clusters reside in higher density environment than unimodal clusters. Clusters in superclusters have higher probability to have substructure than isolated clusters. The superclusters can be divided into two main morphological types, spiders and filaments. Clusters in superclusters of spider morphology have higher probabilities to have substructure and larger peculiar velocities of their main galaxies than clusters in superclusters of filament morphology. The most luminous clusters are located in the high-density cores of rich superclusters. Five of seven most luminous clusters, and five of seven most multimodal clusters reside in spider-type superclusters; four of seven most unimodal clusters reside in filament-type superclusters.

Conclusions. Our study shows the importance of the role of superclusters as high density environment, which affects the properties of galaxy systems in them.
\end{abstract}

Key words. large-scale structure of Universe - galaxies: clusters: general

\section{Introduction}

Most galaxies in the Universe are located in groups and clusters of galaxies, which themselves reside in larger systems - in superclusters of galaxies or in filaments crossing underdense regions between superclusters (Jõeveer et al. 1978; Zeldovich et al. 1982; Oort 1983; de Lapparent et al. 1986). Cluster studies, in combination with the study of their environment are needed to understand the physics of clusters themselves, and the evolution of structure in the Universe.

In the $\Lambda \mathrm{CDM}$ concordance cosmological model groups and clusters of galaxies and their filaments are created by density perturbations of scale up to $32 h^{-1} \mathrm{Mpc}$, and superclusters of galaxies by larger perturbations, up to $100 \mathrm{~h}^{-1} \mathrm{Mpc}$ (Einasto et al. 2011b; Suhhonenko et al. 2011). Still larger perturbations

$\star$ Tables 5, 6, and Appendices are available in electronic form at http://www. aanda.org modulate the richness of galaxy systems. Superclusters of galaxies are the largest density enhancements in the cosmic web. Studies of their properties and galaxy and cluster content are needed to understand the formation, evolution, and properties of the large-scale structure and to compare cosmological models with observations (Kolokotronis et al. 2002; Einasto et al. 2007a,c; Hoffman et al. 2007; Araya-Melo et al. 2009; Einasto et al. 2011e; Sheth \& Diaferio 2011; Lim \& Lee 2012, and references therein).

The structures forming the cosmic web grow by hierarchical clustering driven by gravity (see, e.g., Loeb 2002, 2008, and references therein). Galaxy clusters form at intersections of filaments, through them galaxies and galaxy groups merge with clusters. An indicator of former or ongoing mergers in groups and clusters of galaxies is their multimodality: the presence of a substructure (several galaxy associations within clusters), a large peculiar velocities of their main galaxies, and 
non-Gaussian velocity distribution of their galaxies (Bird \& Beers 1993; Pinkney et al. 1996; Solanes et al. 1999; Knebe \& Müller 2000; Burgett et al. 2004; Flin \& Krywult 2006; Boschin et al. 2008; Andrade-Santos et al. 2012; Hou et al. 2012; Einasto et al. 2012, hereafter E12). More references can be found in E12.

Several studies have shown that richer and more luminous groups and clusters of galaxies from observations and simulations are located in a higher density environment (Einasto et al. 2003a,b, 2005; Croft et al. 2011; Pompei \& Iovino 2012, and references therein). Plionis \& Basilakos (2002) and Plionis (2004) showed that dynamically younger clusters are more strongly clustered than overall cluster population.

In this study we analyse the relations between the multimodality of rich clusters from the SDSS DR8 and the environment where they reside. We calculate the luminosity density field to trace the supercluster-void network, to define the values of the environmental density around clusters, and to determine superclusters of galaxies. For each cluster we find whether the cluster is located in a supercluster and study the relations between the properties of superclusters and clusters. We compare the properties of isolated clusters, and clusters in superclusters, and compare the properties of clusters in superclusters of different morphology, to understand whether the morphology of superclusters is also an important environmental factor in shaping the properties of groups and clusters in superclusters.

E12 analysed the substructure and velocity distributions of galaxies in the richest clusters from the SDSS DR8 with at least 50 member galaxies using a number of tests of different dimensionality. They showed that two most sensitive tests for the presence of substructure were 3D normal mixture modelling and the Dressler-Shectman (DS or $\Delta$ ) test (see also comments in Pinkney et al. 1996; Hou et al. 2012, about the sensitivity of various tests). In this study we use the results of these two tests as an indicators of cluster substructure, and the peculiar velocities of the main galaxies in clusters. With principal component analysis we study the relation between the multimodality of clusters and their environment characterised by the values of the environmental density and supercluster luminosities. In Sect. 2 we describe the data we used, in Sect. 3 we give the results. We discuss the results and draw conclusions in Sect. 4.

We assume the standard cosmological parameters: the Hubble parameter $H_{0}=100 \mathrm{~h} \mathrm{~km} \mathrm{~s}^{-1} \mathrm{Mpc}^{-1}$, the matter density $\Omega_{\mathrm{m}}=0.27$, and the dark energy density $\Omega_{\Lambda}=0.73$.

\section{Data}

We use the MAIN galaxy sample of the 8th data release of the Sloan Digital Sky Survey (Aihara et al. 2011) with the apparent $r$ magnitudes $r \leq 17.77$, and the redshifts $0.009 \leq z \leq 0.200$, in total 576493 galaxies. We corrected the redshifts of galaxies for the motion relative to the $\mathrm{CMB}$ and computed the comoving distances (Martínez \& Saar 2002) of galaxies. The absolute magnitudes of galaxies were determined in the $r$-band $\left(M_{r}\right)$ with the $k$-corrections for the SDSS galaxies, calculated using the KCORRECT algorithm (Blanton \& Roweis 2007). In addition, we applied evolution corrections, using the luminosity evolution model of Blanton et al. (2003). The magnitudes correspond to the rest-frame at the redshift $z=0$. The details about data reduction and the description of the group catalogue can be found in Tempel et al. (2012).

We determine groups of galaxies using the Friends-ofFriends cluster analysis method introduced in cosmology by Turner \& Gott (1976), Zeldovich et al. (1982), Huchra \& Geller (1982). A galaxy belongs to a group of galaxies if this galaxy has at least one group member galaxy closer than a linking length. In a flux-limited sample the density of galaxies slowly decreases with distance. To take this selection effect into account properly when constructing a group catalogue from a flux-limited sample, we rescaled the linking length with distance, calibrating the scaling relation by observed groups (see Tago et al. 2008, 2010, for details). As a result, the maximum sizes in the sky projection and the velocity dispersions of our groups are similar at all distances.

We use in this study systems from the group catalogue with at least 50 member galaxies analysed for substructure in E12. These clusters are chosen from the distance interval $120 h^{-1} \mathrm{Mpc} \leq D \leq 340 h^{-1} \mathrm{Mpc}$ (the redshift range $0.04<$ $z<0.12$ ) where the selection effects are the smallest (we discuss the selection effects in detail in E12 and in Tago et al. 2010). This sample of 109 clusters includes all clusters from the SDSS DR8 with at least 50 member galaxies in this distance interval. E12 showed that more than $80 \%$ of clusters in this sample demonstrate a signs of multimodality according to several $3 \mathrm{D}$, 2D, and 1D tests: the presence of multiple components, large probabilities to have a substructure, and the deviations of galaxy velocity distributions in clusters from Gaussianity. The larger the dimensionality of the test, the more sensitive it is to the presence of substructure in clusters (for details we refer to E12). In this study we use the results of two 3D test to characterise the multimodality in clusters: the 3D normal mixture modelling and the Dressler-Shectman (DS) test. We describe these tests in Appendix A. In addition, we use the peculiar velocity of the main galaxies, $V_{\text {pec }}$. In the group catalogue the main galaxy of a group is defined as the most luminous galaxy in the $r$-band. We use this definition also in the present paper.

We calculate the galaxy luminosity density field to reconstruct the underlying luminosity distribution, and to find the environmental density around clusters. Environmental densities are important for understanding the influence of the local and/or global environment on cluster properties. Three smoothing lengths are used for environmental densities around clusters, 4, 8, and $16 h^{-1} \mathrm{Mpc}$ to characterise environment at scales around clusters from cluster local surroundings to supercluster scales. For details we refer to Appendix B and to Tempel et al. (2012).

To determine supercluster membership for clusters, we first found superclusters (extended systems of galaxies) in the luminosity density field at smoothing length $8 h^{-1} \mathrm{Mpc}$. We created a set of density contours by choosing a density threshold and defined connected volumes above a certain density threshold as superclusters. In order to choose proper density levels to determine individual superclusters, we analysed the density field superclusters at a series of density levels. As a result we used the density level $D=5.0$ (in units of mean density, $\ell_{\text {mean }}=1.65 \times 10^{-2}$ $\left.\frac{10^{10} h^{-2} L_{\odot}}{\left(h^{-1} \mathrm{Mpc}\right)^{3}}\right)$ to determine individual superclusters. At this density level superclusters in the richest chains of superclusters in the volume under study still form separate systems; at lower density levels they join into huge percolating systems. At higher threshold density levels superclusters are smaller and their number decreases.

Superclusters are characterised by their total luminosity, richness, and morphology, determined with Minkowski functionals. The total luminosity of the superclusters $L_{\mathrm{scl}}$ is calculated as the sum of weighted galaxy luminosities:

$L_{\mathrm{scl}}=\sum_{\mathrm{gal} \in \mathrm{scl}} W_{L}\left(d_{\mathrm{gal}}\right) L_{\mathrm{gal}}$. 

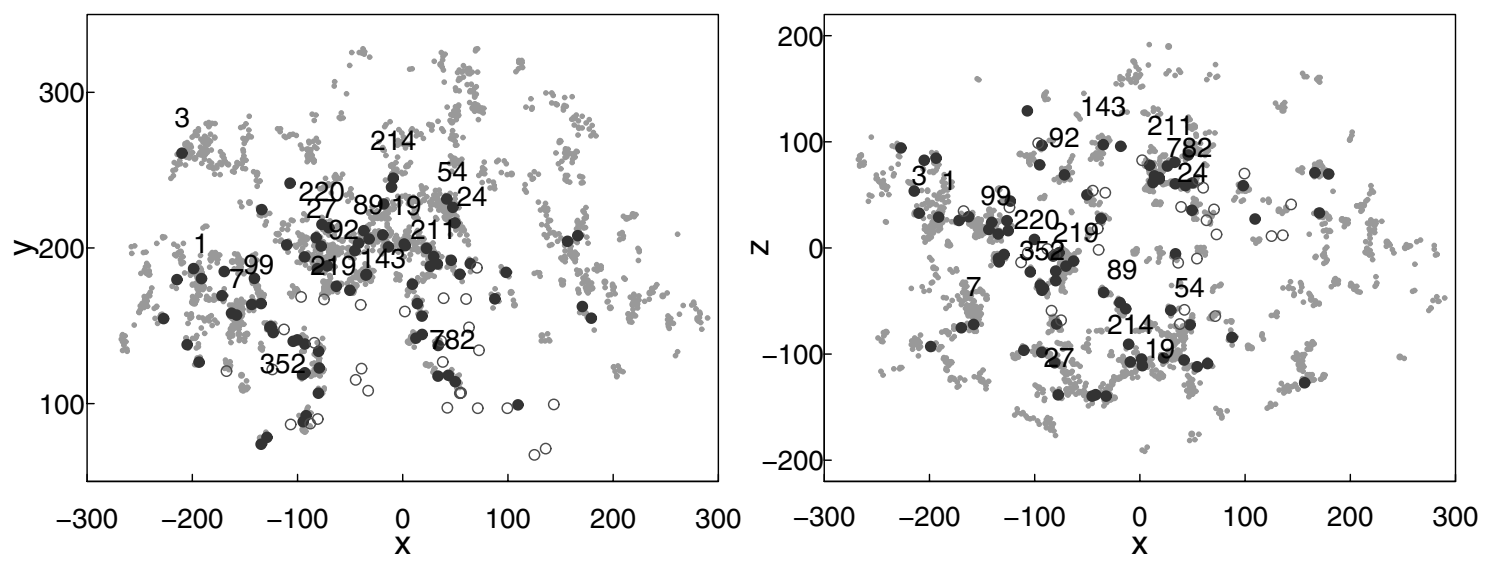

Fig. 1. Distribution of groups with at least four member galaxies in superclusters in $x, y$, and $z$ coordinates (in $h^{-1} \mathrm{Mpc}$, grey dots). Black filled circles denote clusters with at least 50 member galaxies in superclusters, dark grey empty circles those clusters with at least 50 member galaxies which are not located in superclusters. Numbers are ID numbers of superclusters with at least 500 member galaxies.

Here the $W_{L}\left(d_{\text {gal }}\right)$ is the distance-dependent weight of a galaxy (the ratio of the expected total luminosity to the luminosity within the visibility window). The description of the supercluster catalogues is given in Liivamägi et al. (2012) and in Liivamägi et al. (in prep., DR8 catalogue).

The supercluster morphology is fully characterised by the four Minkowski functionals $V_{0}-V_{3}$. For a given surface the four Minkowski functionals (from the first to the fourth) are proportional to the enclosed volume $V$, the area of the surface $S$, the integrated mean curvature $C$, and the integrated Gaussian curvature $\chi$ (Sahni et al. 1998; Martínez \& Saar 2002; Shandarin et al. 2004; Saar et al. 2007; Saar 2009). We give formulaes in Appendix C.

The overall morphology of a supercluster is described by the shapefinders $K_{1}$ (planarity) and $K_{2}$ (filamentarity), and their ratio, $K_{1} / K_{2}$ (the shape parameter), calculated using the first three Minkowski functionals. They contain information both about the sizes of superclusters and about their outer shape. The smaller the shape parameter, the more elongated a supercluster is.

The maximum value of the fourth Minkowski functional $V_{3}$ (the clumpiness) characterises the inner structure of the superclusters and gives the number of isolated clumps, the number of void bubbles, and the number of tunnels (voids open from both sides) in the region (see, e.g. Saar et al. 2007). The larger the value of $V_{3}$, the more complicated the inner morphology of a supercluster is; superclusters may be clumpy, and they also may have holes or tunnels in them (Einasto et al. 2007c, 2011e). Superclusters show large morphological variety in which Einasto et al. (2011d) determined four main morphological types: spiders, multispiders, filaments, and multibranching filaments. Spiders and multispiders are systems of one or several high-density clumps with a number of outgoing filaments connecting them. The Local Supercluster is an example of a typical poor spider. Filaments and multibranching filaments are superclusters with filament-like main body which connects clusters. An example of an exceptionally rich and dense multibranching filament is the richest supercluster in the Sloan Great Wall (Einasto et al. 2007c, 2011e). For simplicity, in this study we classify superclusters as spiders and filaments.

Data about clusters and superclusters are given in Tables 5 and 6. We cross-identify groups with Abell clusters (Table 5). We consider a group identified with an Abell cluster, if the distance between their centres is smaller than at least the linear radius of one of the clusters, and the distance between their centres in the radial (line-of-sight) direction is less than $600 \mathrm{~km} \mathrm{~s}^{-1}$ (an empirical value). In some cases one group can be identified with more than one Abell cluster and vice versa (for details we refer to E12). In Table 6 we give to superclusters the ID number from Einasto et al. (2001) catalogue if there is at least one Abell cluster in common between this catalogue and the present supercluster sample. A common cluster does not always mean that superclusters can be fully identified with each other. A number of superclusters from E01 are split between several superclusters in our present catalogue, an examples of such systems are $\mathrm{SCl} 019$ and $\mathrm{SCl}$ 054, which both belong to SCl 111 in Einasto et al. (2001) catalogue.

\section{Results}

\subsection{The large-scale environment of clusters}

To study the distribution of clusters in the supercluster-void network we present in Fig. 1 the distribution of clusters with at least four member galaxies in superclusters, and the distribution of isolated clusters with at least 50 member galaxies in cartesian coordinates $x, y$, and $z$ defined as in Park et al. (2007) and in Liivamägi et al. (2012):

$$
\begin{aligned}
& x=-d \sin \lambda, \\
& y=d \cos \lambda \cos \eta, \\
& z=d \cos \lambda \sin \eta,
\end{aligned}
$$

where $d$ is the comoving distance, and $\lambda$ and $\eta$ are the SDSS survey coordinates. In Fig. 2 we plot the values of the environmental density around groups with at least 4 member galaxies at smoothing length $8 \mathrm{~h}^{-1}$ Mpc vs. the distance of groups. In this figure circles represent clusters with at least 50 member galaxies. The size of circles is proportional to the number of components in clusters determined with the 3D normal mixture modelling.

Figure 1 (left panel) shows that at the smallest distances from us (at low $y$ values, up to distances approximately $180 \mathrm{~h}^{-1} \mathrm{Mpc}$ ) the sample crosses the void region. This is the void between the nearby rich superclusters (the Hercules supercluster and other systems, the detailed description of the large-scale distribution of superclusters in this region was given in Einasto et al. 2011d). Groups and clusters form two filaments of poor superclusters and isolated clusters crossing this void. The richest superclusters in these filaments are $\mathrm{SCl} 352$ and $\mathrm{SCl} 782$ (we identify 


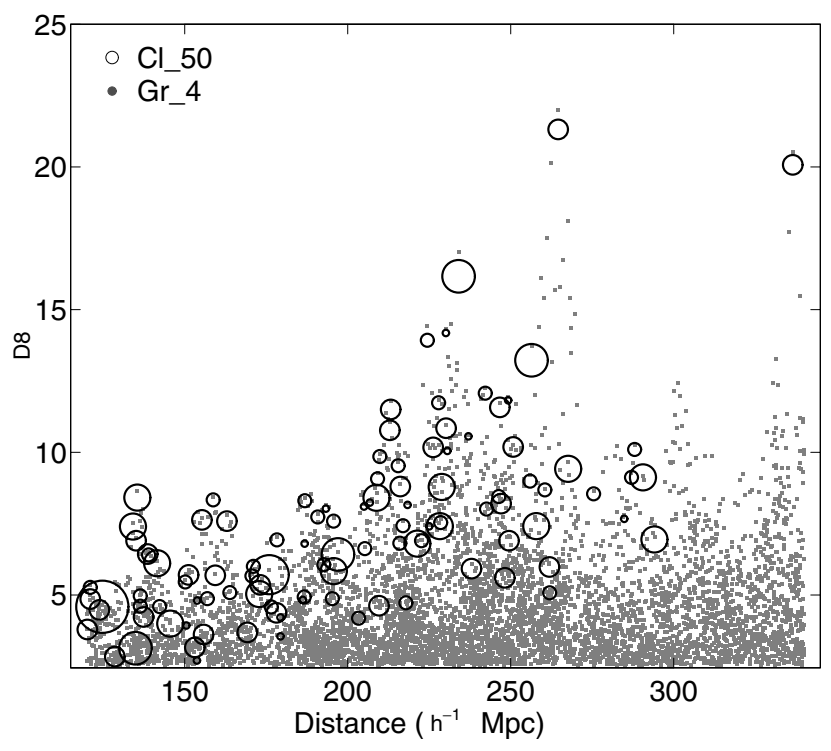

Fig. 2. Global densities at smoothing length $8 h^{-1} \mathrm{Mpc}$ (in units of mean density) around groups and clusters vs. their distance. Black circles denote clusters with at least 50 member galaxies, the size of circles is proportional to the number of components found by $3 \mathrm{D}$ normal mixture modelling. Grey dots denote groups with 4-49 member galaxies.

supercluster members among clusters in Sect. 3.2). The density distribution in Fig. 2 shows that even the maximal values of the environmental densities in this region are low, up to $D 8 \approx 5$, in the density peaks in filaments at the locations of superclusters $D 8<8$ (in units of the mean density). Figures 2 and 1 shows that rich clusters in superclusters mark the peaks in the density distribution, isolated clusters are located at lower densities in filaments. The sizes of symbols in Fig. 2 show that among these clusters there are both multicomponent and onecomponent clusters.

At distances between $180 h^{-1} \mathrm{Mpc}$ and $270 h^{-1} \mathrm{Mpc}$ the SDSS survey crosses systems of rich superclusters. The richest superclusters in these systems are $\mathrm{SCl} 027$ and $\mathrm{SCl} 019$ in the Sloan Great Wall, SCl 211 (the Ursa Major supercluster) in another chain of superclusters, and SCl 099 (the Corona Borealis supercluster) and $\mathrm{SCl} 001$ in the dominant supercluster plane at the intersection of the supercluster chains (for details see Einasto et al. 1997, 2011d). The values of the environmental densities $D 8<8$ in the foreground of rich superclusters at distances less than $200 h^{-1} \mathrm{Mpc}$. At larger distances, in rich superclusters the maximal values of the environmental densities are much higher than in the void region behind them. Again rich clusters mark the high density peaks in the density field (Fig. 2). Some rich clusters are located in the cores of rich superclusters with the highest values of the environmental densities, $D 8>10$ (Einasto et al. 2007b; Tempel et al. 2009, 2011). The environmental density is the largest $(D 8=21.3)$ in the supercluster $\mathrm{SCl} 001$, around rich cluster 29587 (Abell cluster A2142). At still larger distances the SDSS sample reaches the void region behind the Sloan Great Wall and other rich superclusters, and the values of the environmental densities are lower again. The farthest rich cluster in our sample belong to the rich supercluster $\mathrm{SCl} 003$ behind this void at a distance of $336 h^{-1} \mathrm{Mpc}$. Here the value of the environmental density is also very high, $D 8=20.1$.

Figure 2 shows that the lowest values of the environmental densities around rich clusters slightly increase with distance. This is due to the use of groups with 50 and more members. Due to the flux-limited sample, the groups with the same richness are also brighter further away. In E12 we showed that the richness of rich clusters in our sample does not depend on distance, therefore our sample of clusters is not strongly affected by this selection effects. When comparing the environmental densities around clusters in some cases we shall use two distance intervals, to analyse densities and the properties of clusters in void region and in supercluster region separately, and to minimise the influence of this selection effect.

A visual inspection of Fig. 2 shows that both multicomponent and one-component clusters are located in all density peaks. Next we analyse the values of the environmental densities around clusters in more detail.

We plot cluster luminosities vs. environmental densities at three smoothing lengths in Fig. 3, and search for the pairwise correlations between the parameters of clusters and the environmental densities around them with the Pearson's correlation test (Table 1). In Fig. 3 we mark those clusters which are unimodal according to all the tests applied in E12 with filled circles, and those which are multimodal with stars (we discuss them in detail in Sect. 3.3).

We exclude from this analysis the cluster Gr1573 near the edge of the survey for which environmental densities cannot be determined reliably $(D=-999$ in Table 5).

At the smoothing length $4 h^{-1} \mathrm{Mpc}$ the luminosity density is determined by cluster members, and galaxies and galaxy systems in the close neighbourhood of clusters. Therefore the correlation between the luminosities of clusters and environmental densities is strong (the correlation coefficient is $r=0.91$ with very high statistical significance, Table 1). Figure 3 shows that densities around clusters of the same luminosity may differ up to 1.5-2 times depending on the systems around clusters. At this smoothing length there is no statistically significant correlation between the number of components in clusters and the environmental density around clusters. The statistical significance to have substructure in clusters according to the DS test $p_{\Delta}$ is weakly anticorrelated with the environmental density. Small $p_{\Delta}$ values show higher significance of having substructure, therefore this test shows that there is a tendency that clusters with higher probabilities of having a substructure reside in a higher density environments. However, Table 1 shows that the statistical significance of this result is low. Figure 3 shows that one of the most luminous clusters have relatively low density local environment around it (Gr34727 in the supercluster SCl 7, Table D.1).

At the smoothing length $8 h^{-1} \mathrm{Mpc}$ (Fig. 3, middle panel) the scatter of the relation between cluster luminosities and environmental densities increases - the difference between the environmental densities around clusters of the same luminosity increases and the correlations between the cluster luminosities and environmental density become weaker. The scatter is espacially large at densities $D>8$, in the cores of rich superclusters, where both high- and low-luminosity clusters reside. All most luminous clusters are located in supercluster cores. In poor superclusters environmental densities are lower. At the largest smoothing length, $16 h^{-1} \mathrm{Mpc}$ which characterises large scale supercluster environment around clusters the scatter of the relation between cluster luminosities and environmental densities increases and the correlations between the clusters luminosities and environmental density become weaker. The number of components in clusters at large smoothing lengths is not correlated with the environmental density around clusters, the correlation between the probability to have substructure in clusters and environmental density also becomes weaker. The correlations between the peculiar velocities of the main galaxies in clusters and environmental density are statistically highly significant - clusters in 


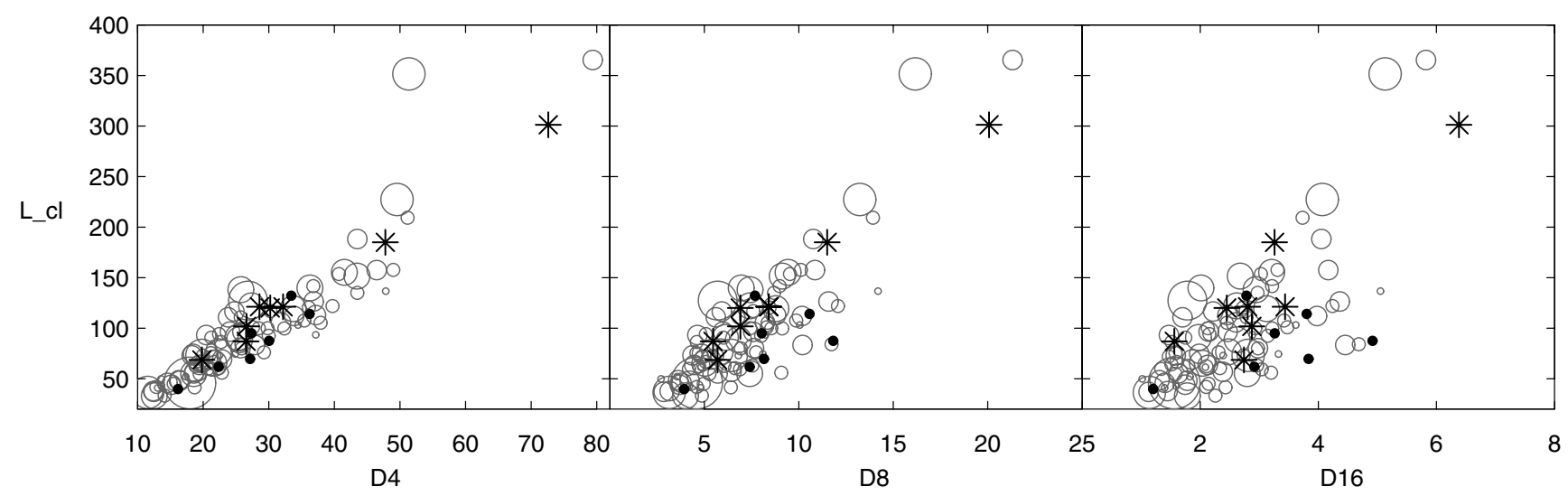

Fig. 3. From left to right: cluster luminosities (in $10^{10} h^{-2} L_{\odot}$ ) vs. their environmental densities at the smoothing lengths 4,8 , and $16 h^{-1} \mathrm{Mpc}$ (in units of mean density) (grey circles). The size of circles is proportional to the number of components found by 3D normal mixture modelling. Stars denote most multimodal clusters, and filled circles denote most unimodal clusters as described in the text.

Table 1. Correlations between the environmental density around clusters, and cluster parameters.

\begin{tabular}{lcccccc}
\hline \hline Parameter & \multicolumn{2}{c}{ D4 } & \multicolumn{2}{c}{ D8 } & \multicolumn{2}{c}{ D16 } \\
& $r$ & $p$ & $r$ & $p$ & $r$ & $p$ \\
\hline$L_{\text {cl }}$ & 0.91 & $1 \mathrm{e}-16$ & 0.85 & $1 \mathrm{e}-16$ & 0.70 & $1 \mathrm{e}-16$ \\
$N_{\text {gal }}$ & 0.59 & $2.7 \mathrm{e}-11$ & 0.54 & $1.8 \mathrm{e}-09$ & 0.42 & $6.2 \mathrm{e}-06$ \\
& & & & & & \\
$N_{\text {comp }}$ & 0.06 & 0.55 & 0.01 & 0.91 & -0.04 & 0.65 \\
$V_{\text {pec }}$ & 0.24 & 0.01 & 0.19 & 0.04 & 0.18 & 0.07 \\
$p_{\Delta}$ & -0.14 & 0.16 & -0.08 & 0.39 & -0.05 & 0.57 \\
& & & & & & \\
$L_{\text {scl }}$ & 0.49 & $7.2 \mathrm{e}-08$ & 0.63 & $4.6 \mathrm{e}-13$ & 0.73 & $2 \mathrm{e}-16$ \\
\hline
\end{tabular}

Notes. Pearson's correlation coefficients $r$ and their $p$-values for correlations between the environmental densities $D 4, D 8$, and $D 16$ around clusters, and the number of 3D components, peculiar velocities of main galaxies, the significance to have substructure according to the DS test, $p_{\Delta}$, the luminosity of clusters, $L_{\mathrm{cl}}$, the numbers of galaxies, $N_{\mathrm{gal}}$, and the total luminosity of superclusters.

higher density environments have larger peculiar velocities of the main galaxies. The correlation coefficients are not large, from 0.24 at smoothing length $4 h^{-1} \mathrm{Mpc}$ to 0.18 at smoothing length $16 h^{-1} \mathrm{Mpc}$. The correlations between the number of galaxies in clusters and the environmental density of clusters are statistically highly significant (Table 1). The correlations are not very strong, with the correlation coefficient $r \approx 0.5$, being stronger at small smoothing length and weaker at large smoothing length. In addition, the larger the smoothing length, the stronger are the correlations between the luminosity of superclusters and environmental density - richer and more luminous superclusters have also higher environmental densities, as found earlier by Einasto et al. (2007a).

Figure 4 shows the cumulative distributions of the values of the environmental densities at smoothing length $4 h^{-1} \mathrm{Mpc}$, at which the correlations between the environmental density and multimodality parameters were the strongest, for two distance intervals: $120 h^{-1} \mathrm{Mpc} \leq D \leq 180 h^{-1} \mathrm{Mpc}$ (upper row), and $180 h^{-1} \mathrm{Mpc} \leq D \leq 300 h^{-1} \mathrm{Mpc}$ (lower row). There are 42 clusters in the closer distance interval, and 65 clusters in the farther distance interval. We show the cumulative distributions of densities around clusters divided into populations according to the different indicators of multimodality: multicomponent and one-component clusters according to the 3D normal mixture modelling, clusters with and without significant substructure according to the DS test $\left(p_{\Delta}<=0.05\right.$, and $p_{\Delta}>0.05$, correspondingly), and clusters with small and large peculiar velocities of their main galaxies. E12 showed that, approximately, the peculiar velocity limit between these two populations is of about $250 \mathrm{~km} \mathrm{~s}^{-1}$. Figure 4 shows that densities around multicomponent clusters and clusters with significant substructure have higher values than densities around one-component clusters without significant substructure. The differences between the densities around clusters with small and large peculiar velocities of their main galaxies in the void region $\left(120 h^{-1} \mathrm{Mpc} \leq\right.$ $D \leq 180 h^{-1} \mathrm{Mpc}$ ) are small, in the farther region in superclusters clusters with large peculiar velocities of their main galaxies have higher environmental densities around them than clusters with small values of the peculiar velocities of their main galaxies.

The relations between the parameters of clusters, the indicators of substructure, and the environmental parameters of clusters can be studied simultaneously with the principal component analysis (PCA). The PCA transforms the data to a new coordinate system, where the greatest variance by any projection of the data lies along the first coordinate (the first principal component), the second greatest variance - along the second coordinate, and so on. There are as many principal components as there are parameters, but often only the first few are needed to explain most of the total variation. The principal components $\operatorname{PC} i(i \in \mathbb{N}$, $i \leq N_{\text {tot }}$ ) are linear combinations of the original parameters:

$P C i=\sum_{k=1}^{N_{\mathrm{tot}}} a(k)_{i} V_{k}$,

where $-1 \leq a(k)_{i} \leq 1$ are the coefficients of the linear transformation, $V_{k}$ are the original parameters and $N_{\text {tot }}$ is the number of the original parameters. In the analysis the parameters are standardised - they are centred on their means, $V_{k}-\overline{V_{k}}$, and normalised, divided by their standard deviations, $\sigma\left(V_{k}\right)$. E12 used PCA to analyse the relations between the multimodality parameters of clusters and their physical properties. We refer to Einasto et al. (2011c) for the references about applications of the PCA in astronomy.

We include into the calculations the number of components as determined with the $3 \mathrm{D}$ normal mixture modelling, $p_{\Delta}$ showing the probability to have substructure according to the DS test, the peculiar velocity of the main galaxy in clusters, $V_{\text {pec }}$, the number of galaxies in clusters, $N_{\text {gal }}$, the environmental density 

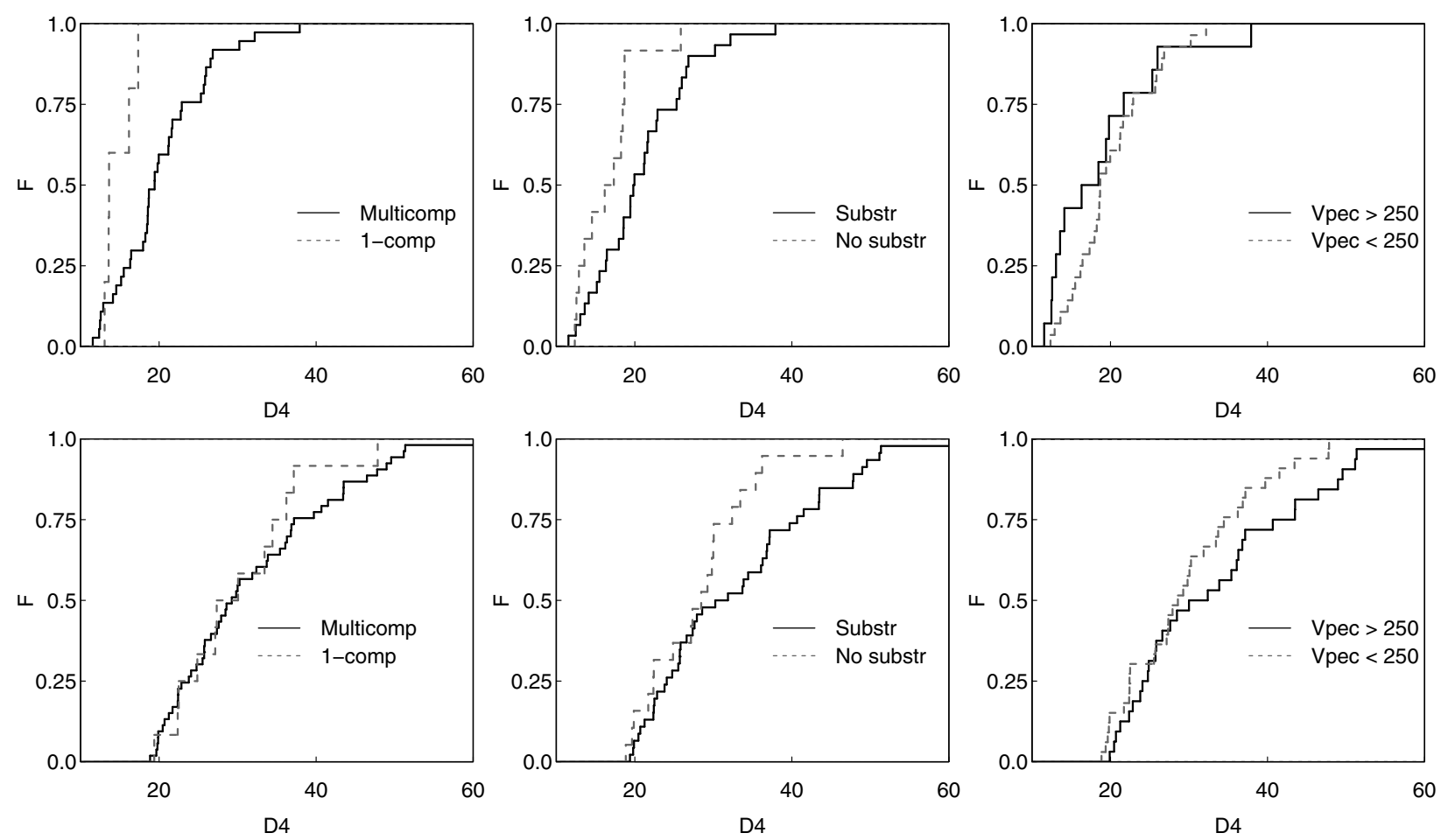

Fig. 4. Cumulative distributions of the values of the environmental densities around clusters for the smoothing length $4 h^{-1} \mathrm{Mpc}$. Solid black line denote densities around multicomponent clusters (left panel), clusters with significant substructure (middle panel), and clusters with the peculiar velocities of their main galaxies larger than $250 \mathrm{~km} \mathrm{~s}^{-1}$ (right panel). Dashed grey line denote densities around one-component clusters (left panel), clusters without significant substructure (middle panel), and clusters with the peculiar velocities of their main galaxies smaller than $250 \mathrm{~km} \mathrm{~s}^{-1}$ (right panel). Upper row - distance interval $120 h^{-1} \mathrm{Mpc} \leq D \leq 180 h^{-1} \mathrm{Mpc}$, lower row - distance interval $180 h^{-1} \mathrm{Mpc} \leq D \leq 300 h^{-1} \mathrm{Mpc}$.

around clusters with smoothing length $8 h^{-1} \mathrm{Mpc}, D 8$ (environmental densities are correlated, therefore we include only one of them), and the luminosity of a supercluster where a cluster resides, $L_{\mathrm{scl}}$. We use $1-p_{\Delta}$ since larger values of $1-p_{\Delta}$ suggest a higher probability to have substructure, therefore the arrows in biplot corresponding to the number of the components point towards the same direction as the arrows corresponding to the DS test. We use logarithms of the peculiar velocities of main galaxies and environmental parameters. Figure 5 and Table 2 show the results of this analysis.

Table 2 shows that the coefficients of the first principal component are the largest for the environmental density around clusters, for the number of galaxies in clusters, and for the total luminosity of superclusters. This shows that richer clusters are located in a higher density environment, and richer superclusters have higher environmental densities in them (Einasto et al. $2007 \mathrm{~b}$,a), as also shown with the analysis above. In the biplot showing the results of the PCA (Fig. 5) the arrows corresponding to the tests about substructure and arrows corresponding to the other parameters of clusters are not pointed into the same direction. This suggests that the correlations between substructure parameters and the environment of clusters are not strong, as also the correlation calculations showed. In Fig. 5 the arrow corresponding to the peculiar velocity of the main galaxies in clusters is pointed approximately into the same direction as the arrow for richness of clusters, showing that these velocities are larger in richer clusters. The length of the arrows and coefficients in Table 2 show that the importance of the peculiar velocity of the main galaxies is smaller than the importance of the richness of clusters.

The first principal component accounts for about $1 / 3$ of the variance of parameters, the second principal component for

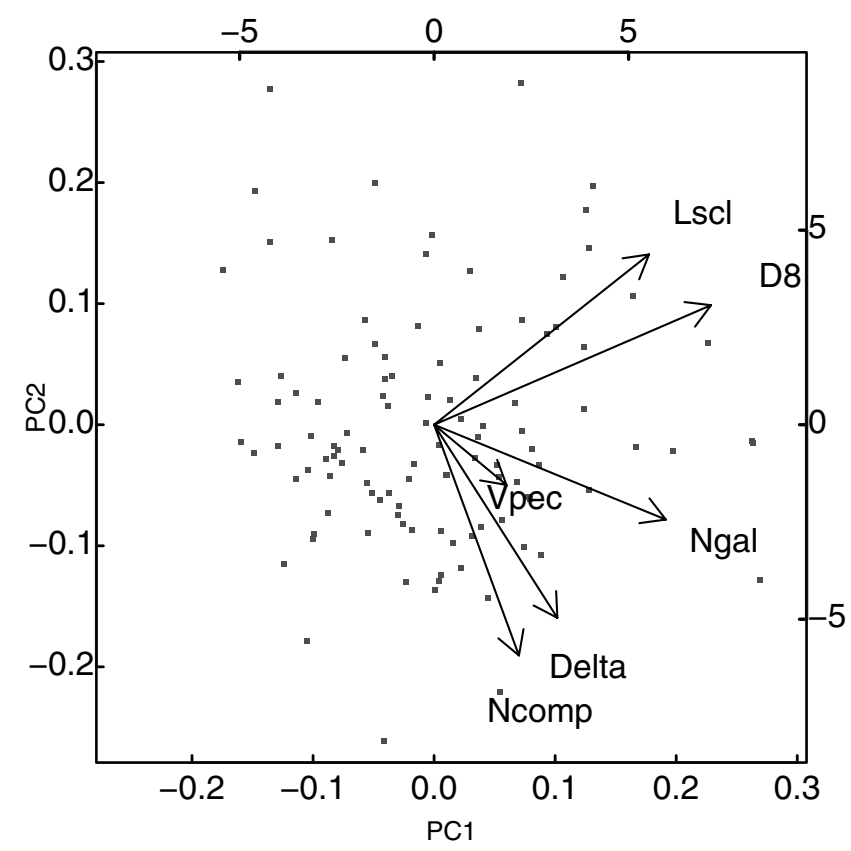

Fig. 5. Biplot of the principal component analysis with the multimodality indicators and the environmental parameters of clusters, as described in the text.

about $1 / 4$ of the variance. However, five principal components are needed to explain more than $90 \%$ of the variance of the parameters, thus clusters with their environment are complicated objects whose properties cannot be explained with a small number of parameters as found also for the dark matter haloes by Jeeson-Daniel et al. (2011). 
Table 2. Results of the principal component analysis for the multimodality and environmental parameters of clusters.

\begin{tabular}{lcccccc}
\hline \hline & PC1 & PC2 & PC3 & PC4 & PC5 & PC6 \\
\hline$N_{\text {comp }}$ & 0.187 & -0.603 & 0.164 & 0.153 & -0.736 & -0.087 \\
$\log \left(V_{\text {pec }}\right)$ & 0.160 & -0.157 & -0.969 & -0.068 & -0.064 & 0.026 \\
$p_{\Delta}$ & 0.272 & -0.504 & 0.148 & -0.703 & 0.380 & -0.094 \\
$\log \left(N_{\text {gal }}\right)$ & 0.512 & -0.247 & 0.070 & 0.555 & 0.412 & 0.437 \\
$\log (D 8)$ & 0.612 & 0.312 & 0.020 & 0.122 & 0.016 & -0.714 \\
$\log \left(L_{\text {scl }}\right)$ & 0.475 & 0.445 & 0.073 & -0.390 & -0.371 & 0.528 \\
\hline Importance of components \\
PC1 dev. & PC1 & PC2 & PC3 & PC4 & PC5 & PC6 \\
Prop. of var. & 0.397 & 1.183 & 0.983 & 0.870 & 0.833 & 0.481 \\
Cum. prop. & 0.325 & 0.233 & 0.161 & 0.126 & 0.116 & 0.039 \\
\hline
\end{tabular}

Notes. St. dev. denotes standard deviation, Prop. of var. denotes proportion of variance, and Cum. prop. denotes cumulative proportion.

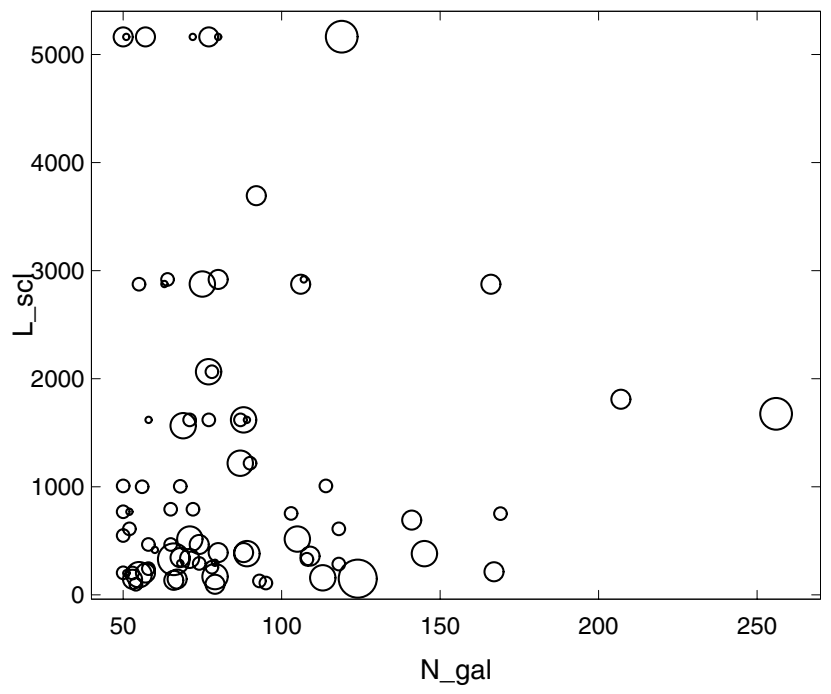

Fig. 6. The number of galaxies in clusters vs. the total luminosity of superclusters where they reside (in $10^{10} h^{-2} L_{\odot}$ ). The size of symbols is proportional to the number of components in clusters.

The locations of clusters in the PC1-PC2 plane shows that unimodal clusters in superclusters are located at upper lefthand part of the plot and have larger PC2 and smaller (larger negative) PC1 values (for example, clusters 608, 13408, 25078, and 28508). Rich multimodal clusters of high environmental density value around them populate lower and middle righthand area of the biplot (clusters 34276, 34727, 914, 29587). Multimodal clusters in low environmental density environment populate the lefthand lower area of the PC1-PC2 plane (clusters 11474, 11015). Unimodal clusters in very rich superclusters populate the upper righthand area of the plane $(67116,63361$, and others). On the lefthand area of the plane are located isolated multimodal poor clusters $(50657,58323$, and others).

In Fig. 6 we show for clusters in superclusters the number of galaxies in clusters vs. the total luminosity of the host supercluster. Here the supercluster of the highest luminosity is SCl 027 , the richest system in the Sloan Great Wall. This figure shows that this supercluster, as well as other superclusters host both multicomponent and one-component clusters, as a result there is no correlation between the host supercluster luminosity and the number of components in clusters.

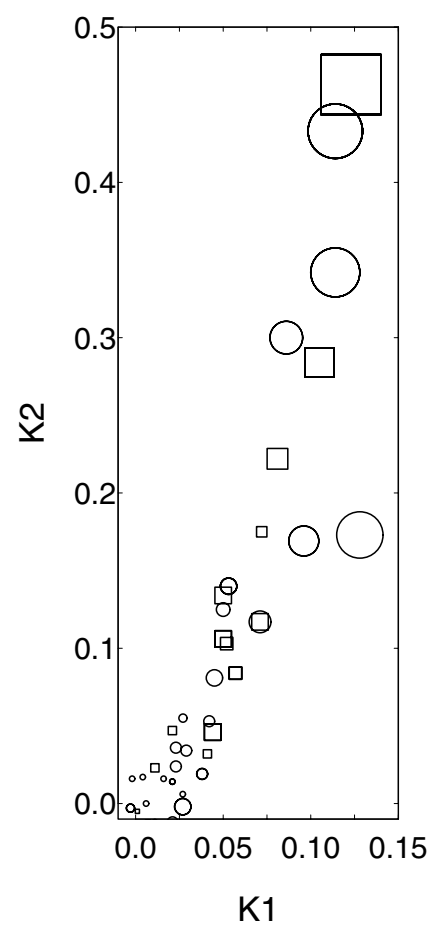

Fig. 7. Shapefinders $K_{1}$ (planarity) and $K_{2}$ (filamentarity) for the superclusters. The symbol sizes are proportional to the fourth Minkowski functional $V_{3}$. Circles denote the superclusters of spider morphology and squares denote the superclusters of filament morphology.

\subsection{Properties of clusters and supercluster morphology}

In this section we analyse the properties of clusters in superclusters of different morphology. At first we searched for the host superclusters for each cluster and found that 80 of our clusters lie in superclusters. Next we determined for these superclusters their morphological parameters and types using Minkowski functionals and shapefinders, and visual inspection. The physical and morphological parameters of superclusters (the values of the fourth Minkowski functional (the clumpiness) $V_{3}$ and the shapefinders $K_{1}$ (the planarity), $K_{2}$ (the filamentarity), and their ratio (the shape parameter) for each supercluster are given in Table 6. According to their overall shape superclusters are elongated with the value of the filamentarity $K_{2}$ being larger than the value of the planarity $K_{1}$. There are only 4 systems with the shape parameter $K_{1} / K_{2}>1.0$ resembling pancakes. The superclusters with very small values of the shapefinders have large negative values of the shape parameter owing to noisiness in the data. There are 15 superclusters of filament morphology, and 35 of spider morphology in our sample. Figure 7 shows the shapefinders plane for the superclusters with the size of symbols proportional to the clumpiness of superclusters takes together the morphological information about superclusters. Superclusters with higher values of planarity and filamentarity have also larger values of clumpiness and therefore more complicated inner morphology. Poor superclusters are mostly of spider morphology (we refer for details about the morphological information to Einasto et al. 2011d). Most of them are located close to us, they are members of the filaments crossing the void region in front of the Sloan Great Wall and other rich superclusters at distances larger than $180 \mathrm{~h}^{-1} \mathrm{Mpc}$.

In Fig. 8 we show the examples of the fourth Minkowski functional $V_{3}$ vs. mass fraction $m_{f}$ and the shapefinders $K_{1}$ and $K_{2}$ for two superclusters of filament morphology, $\mathrm{SCl} 027$ 

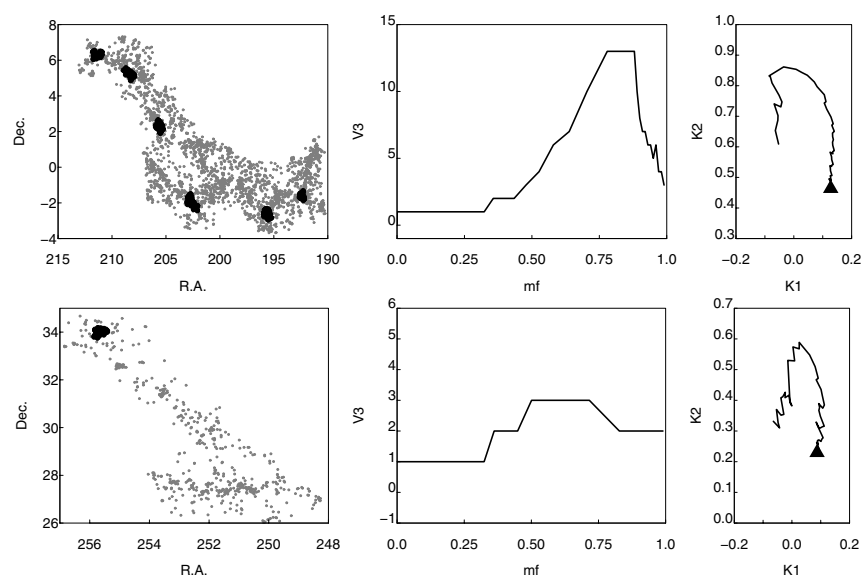

Fig. 8. Sky distribution of galaxies (left panel), the fourth Minkowski functional $V_{3}$ (middle panel) and the shapefinder's $K_{1}-K_{2}$ plane (right panel) for two superclusters of filament morphology. Upper row - the supercluster $\mathrm{SCl}$ 027, lower row - the supercluster SCl 059. In the left panel black filled circles denote galaxies in clusters with at least 50 member galaxies, grey dots denote other galaxies. On the right panel triangle corresponds to $K_{1}$ and $K_{2}$ values at the mass fraction $m_{\mathrm{f}}=0$ (the whole supercluster). Mass fraction increases anti-clockwise along the $K_{1}-K_{2}$ curve (the morphological signature).

and $\mathrm{SCl}$ 059, in Fig. 9 for superclusters of spider morphology, SCl 019 and SCl 092. The superclusters SCl 027 and SCl 019 are the richest two superclusters in the Sloan Great Wall (Einasto et al. 2011e,d). In middle panel of these figures we plot the clumpiness $V_{3}$ vs. the (excluded) mass fraction $m_{f}$. At small mass fractions the isodensity surface includes the whole supercluster and the value of the 4th Minkowski functional $V_{3}=1$. As we move to higher mass fractions, the iso-surfaces move from the outer supercluster boundary towards the higher density parts of a supercluster, and some galaxies do not contribute to the supercluster any more. Individual high density regions in a supercluster begin to separate from each other, also the holes or tunnels may appear, therefore the value of the clumpiness increases. At a certain mass fraction $V_{3}$ has a maximum, showing the largest number of isolated clumps in a given supercluster. At still higher mass fraction only the high density peaks remain in the supercluster and the value of $V_{3}$ decreases again. When we increase the mass fraction, the changes in the morphological signature accompany the changes of the 4th Minkowski functional (right panels of the figures). As the mass fraction increases, at first the planarity $K_{1}$ almost does not change, while the filamentarity $K_{2}$ increases - at higher density levels superclusters become more filament-like than the whole supercluster. Then also the planarity starts to decrease, and at a mass fraction of about $m_{\mathrm{f}}=0.7$ the characteristic morphology of a supercluster changes. We see the crossover from the outskirts of a supercluster to the core of a supercluster (Einasto et al. 2007c). The figures of the fourth Minkowski functional and shapefinders for rich superclusters with at least 300 member galaxies from SDSS DR7 can be found in Einasto et al. (2011d).

Next we compare the properties of clusters in superclusters of filament and of spider morphology, and isolated clusters in two distance intervals. At distances up to approximately $180 \mathrm{~h}^{-1} \mathrm{Mpc}$ most superclusters are poor, and of spider morphology. In this distance interval we compare the properties of isolated clusters and supercluster members, without dividing them according to the host supercluster morphology. At distances of $180 h^{-1} \mathrm{Mpc} \leq D \leq 300 h^{-1} \mathrm{Mpc}$ there are only six isolated
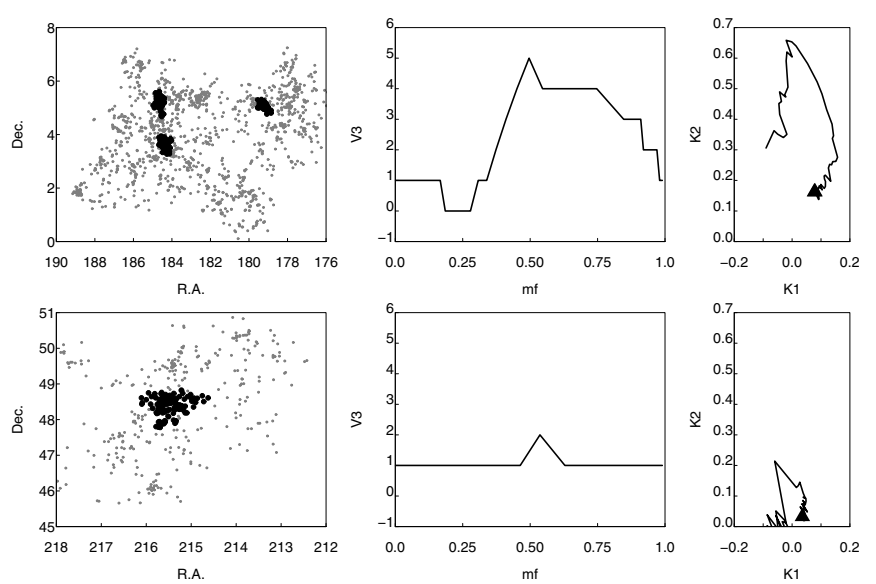

Fig. 9. Sky distribution of galaxies (left panel), the fourth Minkowski functional $V_{3}$ (middle panel) and the shapefinder's $K_{1}-K_{2}$ plane (right panel) for two superclusters of spider morphology. Upper row - the supercluster SCl 019, lower row - the supercluster SCl 092. Notations as in Fig. 8.

clusters, therefore we compare the properties of clusters in superclusters of different morphology. For a comparison we also show parameters of isolated clusters in this distance interval. We present cumulative distributions of the cluster substructure parameters in Fig. 10 and the median values of cluster parameters in Tables 3 and 4.

Table 3 and Fig. 10 (upper row) show that clusters in superclusters of spider morphology have higher probabilities to have substructure, and larger peculiar velocities of their main galaxies than clusters in filament-type superclusters. Clusters in spider-type superclusters are slightly richer than those in filament-type superclusters. Differences in a number of components found by 3D normal mixture modelling are small. The Kolmogorov-Smirnov test with substructure parameters centred on their means showed that in this case the differences between the probalitities to have substructure according to the DS test are statistically of very high significance $\left(p<10^{-6}\right)$, differences between other centred parameters are not significant $(p>0.2)$.

Isolated clusters in this distance interval are poor, even poorer than nearby isolated clusters, with 52 median number of galaxies. The maximal values of the peculiar velocities of their main galaxies, and maximal number of components are smaller than those for clusters in superclusters of both types and smaller than those for nearby isolated clusters, but only one of them is a one-component cluster without significant substructure.

As an example of a nearby supercluster of spider morphology we show in Fig. 11 the distribution of galaxies in SCl 352 in the filament crossing the void between nearby superclusters and the Sloan Great Wall (Fig. 1). This supercluster contain two clusters with at least 50 member galaxies, Gr9350 and Gr34926. Both have four components and high probability to have substructure according to the DS test. The peculiar velocities of their main galaxies are small. In the Gr9350 the main galaxy is located in the main component of the cluster with large rms velocities of galaxies (the finger-of-god, seen in the right panel of the figure). In Gr34926 the main galaxy is located in another component with smaller rms velocities of galaxies. We see in this figure rich inner structure of the supercluster, where clusters and groups of galaxies are connected by galaxy filaments. Some components of rich clusters may be infalling, a hint that clusters are not virialised yet. 

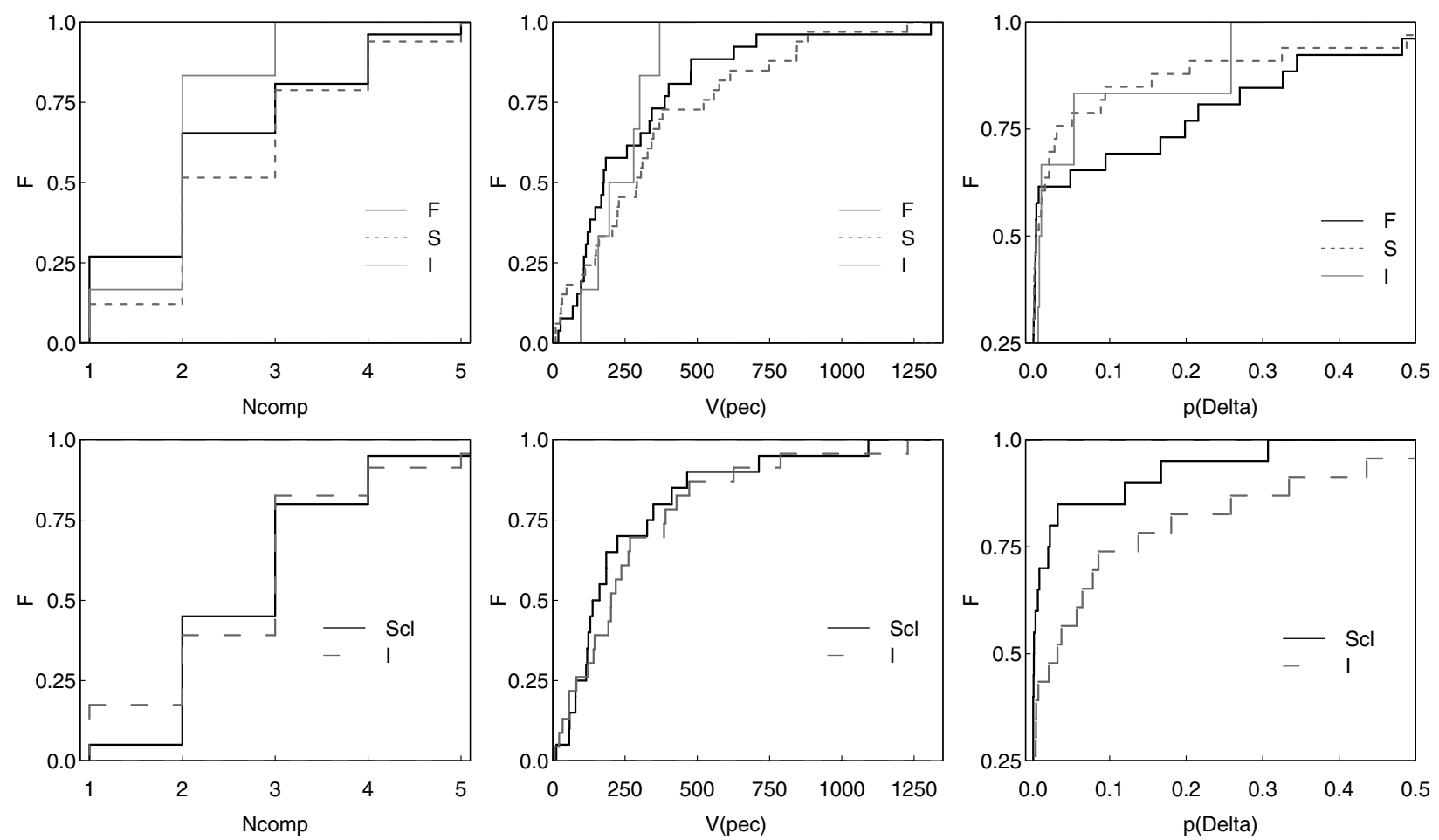

Fig. 10. Cumulative distributions of the numbers of components in clusters, $N_{\text {comp }}$, peculiar velocities of cluster main galaxies, $V_{\text {pec }}$ (in km s ${ }^{-1}$ ), and $p$-value of the DS test, $p_{\Delta}$ for clusters in superclusters of filament morphology (F, black solid line), of spider morphology (S, grey dashed line), and for isolated clusters (I, thin grey solid line) in the distance interval $180 h^{-1} \mathrm{Mpc} \leq D \leq 300 h^{-1} \mathrm{Mpc}$ (upper row), and for clusters in superclusters (Scl, black solid line) and for superclusters in low density regions (isolated clusters I, grey solid line) in the distance interval $120 h^{-1} \mathrm{Mpc} \leq D \leq$ $180 \mathrm{~h}^{-1} \mathrm{Mpc}$ (lower row).

Table 3. Properties of clusters in superclusters of spider and filament morphology.

\begin{tabular}{lcc}
\hline \hline$(1)$ & $(2)$ & $(3)$ \\
Parameter & Spiders & Filaments \\
\hline$N_{\text {cl }}$ & 33 & 26 \\
$N_{\text {gal }}$ & $77 \pm 15$ & $69 \pm 15$ \\
& & \\
$N_{\text {comp }}$ & $2.0 \pm 0.46$ & $2.0 \pm 0.45$ \\
$V_{\text {pec }}$ & $291 \pm 60$ & $176 \pm 55$ \\
$p_{\Delta}$ & $0.004 \pm 0.013$ & $0.004 \pm 0.020$ \\
\hline
\end{tabular}

Notes. Median values and their errors of the number of $3 \mathrm{D}$ components, peculiar velocities of main galaxies (in $\mathrm{km} \mathrm{s}^{-1}$ ), and $p_{\Delta}$, and the numbers of galaxies, $N_{\text {gal }}$ for clusters in superclusters of spider and filament morphology (denoted as spiders and filaments) in a distance interval $180 h^{-1} \mathrm{Mpc} \leq D \leq 300 h^{-1} \mathrm{Mpc}$.

Nearby clusters in superclusters are richer than more distant clusters in superclusters. They have larger probabilities to have substructure than clusters in more distant superclusters, but smaller peculiar velocities of their main galaxies. We use fluxlimited sample of galaxies to define clusters, and nearby clusters contain galaxies of lower luminosity than distant clusters. It is possible that these clusters have substructure in their outer regions formed by fainter galaxies, absent in more distant sample, and this may explain the difference between them. They also have different global environment: nearby superclusters are located in poor filaments surrounded by voids. More distant superclusters are richer and form several chains of rich superclusters (for details about the global environment of superclusters we refer to Einasto et al. 2011d). Next we plan to analyse the galaxy
Table 4. Properties of clusters in superclusters and isolated clusters.

\begin{tabular}{lcc}
\hline \hline$(1)$ & $(2)$ & $(3)$ \\
Parameter & Supercluster members & Isolated clusters \\
\hline$N_{\text {cl }}$ & 20 & 23 \\
$N_{\text {gal }}$ & $84 \pm 20$ & $58 \pm 14$ \\
& & \\
$N_{\text {comp }}$ & $3.0 \pm 0.63$ & $3.0 \pm 0.59$ \\
$V_{\text {pec }}$ & $150 \pm 56$ & $201 \pm 59$ \\
$p_{\Delta}$ & $0.001 \pm 0.008$ & $0.032 \pm 0.020$ \\
\hline
\end{tabular}

Notes. Median values and their errors of the number of 3D components, peculiar velocities of main galaxies (in $\mathrm{km} \mathrm{s}^{-1}$ ), and $p_{\Delta}$, and the numbers of galaxies, $N_{\text {gal }}$ for clusters in superclusters and for isolated clusters in a distance interval $120 h^{-1} \mathrm{Mpc} \leq D \leq 180 \mathrm{~h}^{-1} \mathrm{Mpc}$.

content of clusters in more detail, and also a larger sample of clusters and superclusters, to understand better the difference between clusters.

Isolated clusters are poorer than clusters in superclusters. The number of components in isolated clusters is close to that for supercluster members in the same distance interval. The peculiar velocities of the main galaxies in isolated clusters are larger than in supercluster members, but maximal values of the peculiar velocities of main galaxies in isolated clusters are smaller than those in supercluster members. The DS test shows that the probability to have substructure is larger among nearby supercluster member clusters than in isolated clusters. Among six distant isolated clusters only one has no significant substructure. 

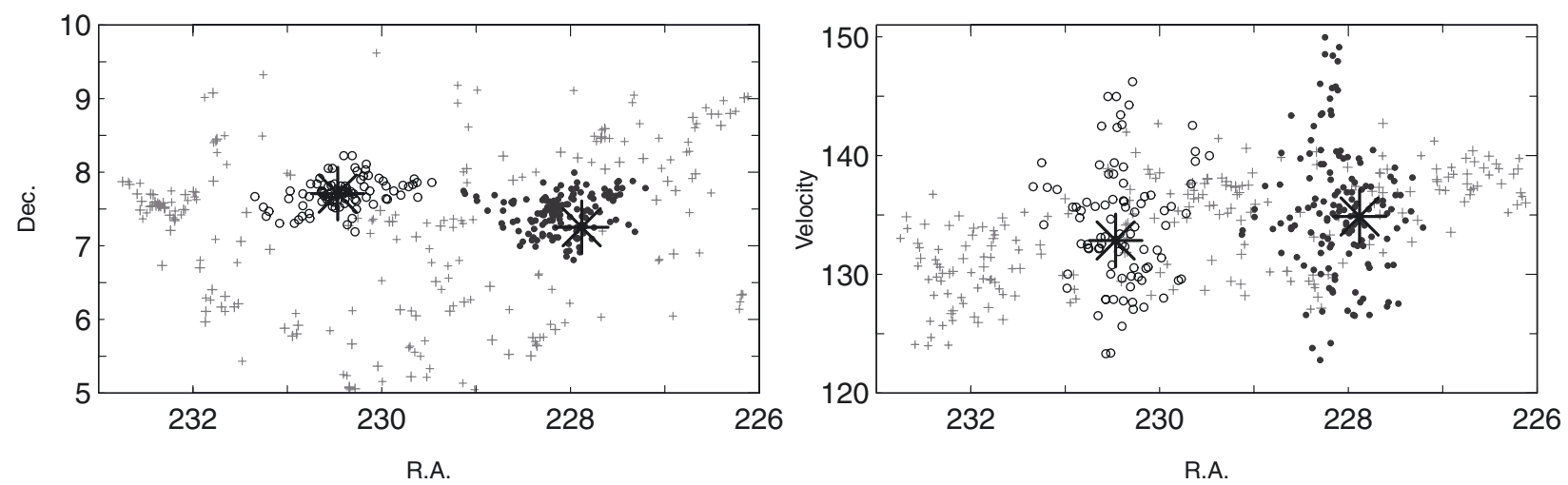

Fig. 11. Distribution of galaxies in RA vs. Dec, and RA vs. velocity (in $10^{2} \mathrm{~km} \mathrm{~s}^{-1}$ ) plot (right panel) in the supercluster SCl 352 . Filled circles denote galaxies in Gr34726, empty circles galaxies in Gr9350. Grey crosses denote other galaxies in the supercluster. Black stars show the location of the main galaxies in both rich cluster.
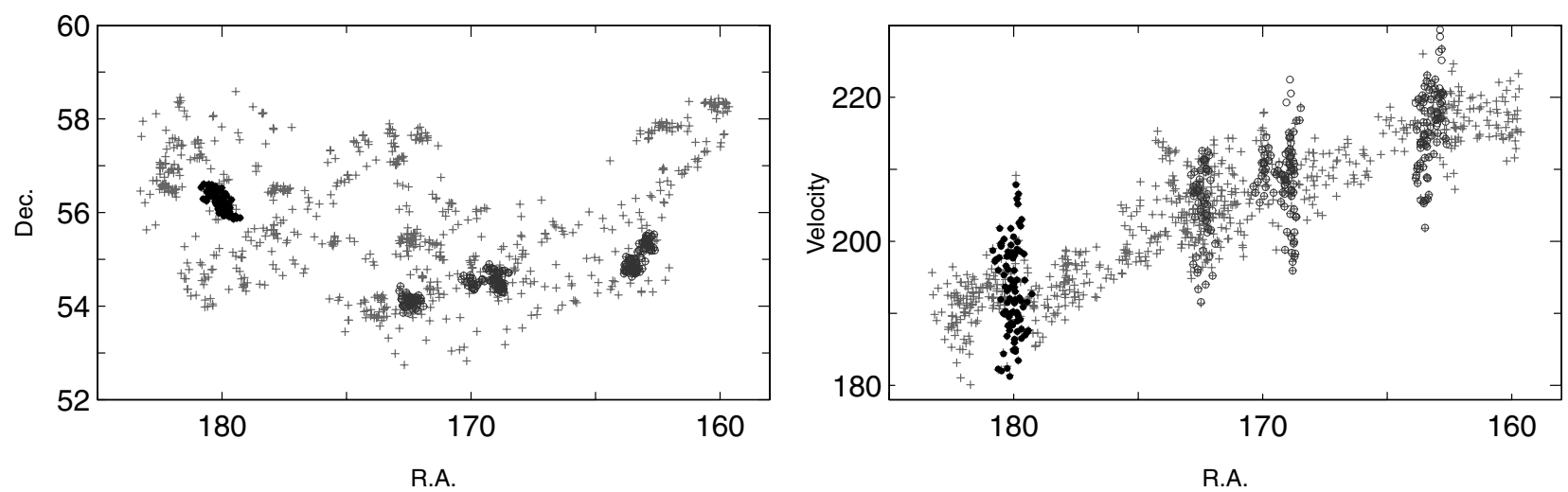

Fig. 12. Distribution of galaxies in RA vs. Dec, and RA vs. velocity (in $10^{2} \mathrm{~km} \mathrm{~s}^{-1}$ ) plot (right panel) in the supercluster SCl 211 (the Ursa Major supercluster). Filled circles denote galaxies in Gr5217 (Abell cluster A 1436), empty circles galaxies in other clusters with at least 50 member galaxies. Grey crosses denote other galaxies in the supercluster.

\subsection{Examples of selected clusters}

Most luminous clusters. Our sample contains seven clusters of very high luminosity (Table D.1). All of them have been identified with Abell clusters. They are located in high density cores of superclusters (Sect. 3.1). Five of seven most luminous clusters are located in superclusters of spider morphology. Among them are the cluster Gr34727 and the cluster Gr39489 in superclusters of the dominant supercluster plane ( $\mathrm{SCl} \mathrm{007,} \mathrm{and}$ SCl 099, the Corona Borealis supercluster, correspondingly). The Corona Borealis supercluster and clusters in it have been studied by Small et al. (1998) and Padilla-Torres et al. (2009). One of the high- luminosity clusters in the filament-type supercluster is Gr914 (Abell cluster A 1750) in the richest supercluster of the Sloan Great Wall, SCl 027, another - Gr29587 (A2142) in $\mathrm{SCl} 001$ in the dominant supercluster plane. The luminosity density around A2142 in SCl 001 is very high (for details and references see Einasto et al. 2011d). The clusters A1750, A2028, A2029, A2065, A2069, and A2142 are (probably merging) X-ray clusters (Markevitch et al. 2000; Belsole et al. 2004; Clarke et al. 2004; Bourdin \& Mazzotta 2008; Owers et al. 2009; Gastaldello et al. 2010). All high- luminosity clusters have multiple components, high probability to have substructure, and most of them have large peculiar velocities of their main galaxies.

Most unimodal and most multimodal clusters. Some of our clusters are unimodal according to all the tests applied in E12, they are one-component systems with very low probability of substructure, the sky distribution of their member galaxies is symmetrical, and the galaxy velocity distribution is Gaussian. We list them in Table D.2. There are also multimodal clusters according to all the tests with multiple components, asymmetrical galaxy distribution and non-Gaussian distribution of galaxy velocities (Table D.3). We marked them in luminosity-density plots in Fig. 3. Tables D.2 and D.3 show that unimodal clusters are, in average, poorer and of lower luminosity than multimodal clusters. Figure 4 showed that unimodal clusters are typically located in a lower density environment, but in Fig. 3 we saw that some of them reside also in high-density cores of rich superclusters. Of seven most unimodal clusters four are located in superclusters of filament morphology, two in spider-type superclusters and one is isolated. In contrary, of seven most multimodal clusters five are located in spider-type superclusers, and only two in filament-type superclusters. There are two superclusters in our sample which both hosts one most multimodal and one most unimodal cluster - SCl 027, and SCl 211. In Fig. 12 we plot the distribution of galaxies in the supercluster $\mathrm{SCl} 211$ (the Ursa Major supercluster). Here Gr5217 is located approximately at $\mathrm{RA}=180$ and $\mathrm{Dec}=56.2$ degrees, and $\mathrm{Gr} 28387$ at $\mathrm{RA}=170$ and $\operatorname{Dec}=54.5$ degrees.

Unimodal cluster Gr25078 (Abell cluster A1650) in the core of $\mathrm{SCl} 027$ is a compact X-ray source, possibly located at a cold spot in the CMB (Udomprasert 2004). The cluster A1809 in the supercluster $\mathrm{SCl} 027$ is also a X-ray cluster. Multimodal 
clusters A1291, A1983 and A671 are also X-ray clusters (Pratt \& Arnaud 2003; Einasto et al. 2011d; Pan et al. 2012).

\section{Discussion and conclusions}

We studied the environment of rich clusters from SDSS DR8, defined by the environmental luminosity density around clusters, and by membership of clusters in superclusters of different morphology. We found a correlation between the environmental density around clusters and the presence of substructure in clusters. However, both multimodal and unimodal clusters can be found in regions of low and high environmental density, and correlations with the environmental density are not strong. In the study of the substructure of the richest clusters of the Sloan Great Wall Einasto et al. (2010) found clusters with substructure in both rich and poor superclusters of the Wall. In this study we showed using a larger sample of superclusters and clusters that superclusters of different richness may host both multicomponent and one-component clusters, and there is no correlation between the host supercluster richness (luminosity) and the multimodality of clusters. In higher density environment the peculiar velocities of the main galaxies in clusters are larger, a suggestion that by this measure also clusters in our sample are dynamically more active in high density environments. Isolated clusters are poorer and have smaller maximal number of components than cluster in superclusters in the same distance interval. Plionis \& Basilakos (2002), Plionis (2004), Ragone-Figueroa \& Plionis (2007) and Espino-Briones et al. (2007) showed that dynamically younger clusters with more substructure are more strongly clustered than overall cluster population. They used cluster centroid shift and DS test results as indicators of the dynamical state of clusters. We only use the data about rich clusters in our sample, and majority of them (80\%) show multimodality according to at least one test in E12 while other studies included data also about poorer systems. This is probably the reason why we found weaker correlations between the presence of substructure and cluster environment than in other studies.

We also found clusters in our sample with almost no close galaxies or galaxy filaments (within the SDSS survey magnitude limits) in the sky distribution. One example of such a cluster is given in Fig. 12. This is the unimodal cluster Gr5217 (A1436), surrounded by an almost empty region without visible galaxies, seen also in Fig. 1b by Kopylova \& Kopylov (2007). Other rich clusters in this system are connected by galaxy filaments. This contradicts with understanding that rich clusters are located at the intersections of galaxy filaments. One possible reason for that may be that all brighter galaxies in this region have already merged into the cluster. Kopylova \& Kopylov (2009) write that A1436 is probably relaxing after a recent merger, which agrees with our interpretation. We found about ten of such clusters in our sample, both one- component and multicomponent ones, and continue to study them to better understand the relation between rich clusters and their small and large scale environment.

Cosmological simulations of the future evolution of the structure in the Universe predict that future superclusters (at $a=100, a$ is the expansion factor) are much more spherical than present-day superclusters. Clusters in superclusters will merge into a single cluster in the far future. In other words, the differences noted in this study between superclusters of different morphology, and clusters in them, may disappear in the distant future (see Araya-Melo et al. 2009, for a review and references).

In the framework of the hierarchical formation of the structure galaxy clusters are located at the intersections of filaments which form the cosmic web. Matter flows through filaments from lower density regions into clusters. The merging and growth of dark matter haloes have been studied with cosmological simulations (Richstone et al. 1992; Mo \& White 2002; McIntosh et al. 2008; Fakhouri et al. 2010, and references therein) which show that the late time formation of the main haloes and the number of recent major mergers can cause the late time subgrouping of haloes and the presence of substructure (Smith \& Taylor 2008; Einasto et al. 2010; Power et al. 2012). In high-density regions groups and clusters of galaxies form early and could be more evolved dynamically (Tempel et al. 2009), but in high-density regions the velocities of haloes in the vicinity of larger haloes are high (Einasto et al. 2005) and the possibility of mergers is also high. As a result in high-density regions clusters have a larger amount of substructure and higher peculiar velocities of their main galaxies than in low-density regions.

Superclusters of spider morphology have richer inner structure than superclusters of filament morphology with large number of filaments between clusters in them. This may lead to the differences noted in this study: clusters in superclusters of spider morphology are dynamically younger than clusters in superclusters of filament morphology with their higher probability to have substructure and larger peculiar velocities of main galaxies. Five of seven most multimodal clusters are located in superclusters of spider morphology, while four of six most unimodal clusters in superclusters reside in filament-type superclusters.

One example of the high-luminosity multimodal cluster in the spider-type supercluster is Gr34727 (Abell cluster A2028). Gastaldello et al. (2010) suggest that the subclusters of A 2028 are probably merging to produce a more massive cluster. Merging X-ray clusters have been found also in the high-density cores of other superclusters (Rose et al. 2002; Bardelli et al. 2000).

Einasto et al. (2011e) calculated the peculiar velocities of the main galaxies in groups from the two richest superclusters in the Sloan Great Wall and found that groups in the supercluster SCl 027 of filamentary morphology (see Table 6) are dynamically more active with their larger peculiar velocities of main galaxies than groups in $\mathrm{SCl} 019$ of spider morphology. In $\mathrm{SCl} 027$ the environmental densities are very high, this may be the reason why in this supercluster of filament morphology clusters show higher dynamical activity than another supercluster of the Sloan Great Wall.

Einasto et al. (2011a,b), and Suhhonenko et al. (2011) showed how the syncronisation of phases of density waves of different scales affect the richness of galaxy systems: the larger the scale of density waves, where the maxima of waves of different scales have close positions, the larger are the masses of galaxy clusters. In lower density regions the formation of rich clusters is suppressed by the combined negative sections of medium- and large-scale density perturbations. This process makes voids empty of galaxies and their systems, clusters of galaxies can be found in filaments crossing the voids, and they are not so rich as galaxy clusters in higher density regions. This is what we found in this study. Richer clusters from our sample are located in regions of high environmental density, in agreement also with earlier results about observations and simulations (Einasto et al. 2003a,b, 2005; Croft et al. 2011, and references therein), where positive sections of medium- and large-scale density perturbations combine. The most luminous clusters are located in high-density cores of rich superclusters, five of seven most luminous clusters are in spider-type superclusters. From the other hand, Einasto et al. (2011d) studied the morphology of superclusters from SDSS DR7 and found that among them there are no compact and very luminous superclusters. Poor 
superclusters have lower luminosities and they host clusters of lower luminosity.

Correlations between the cluster parameters and the environmental density are stronger at small smoothing lengths and become weaker as we increase the smoothing length, in agreement with Einasto et al. (2003a) and Einasto et al. (2003a) who showed that the properties of galaxy groups depend on environmental density up to scales of about $15-20 h^{-1} \mathrm{Mpc}$. Simulations show that also halo spin, overall shape and other properties depend on environment (Einasto et al. 2005; Hahn et al. 2007; White et al. 2010; Noh \& Cohn 2011; Wang et al. 2011; Codis et al. 2012, and references therein) although Jeeson-Daniel et al. (2011) and Skibba \& Macciò (2011) showed that dependence on environment is weaker than found in other studies.

Summarising, our conclusions are as follows.

1) Clusters from our sample are located in density peaks in filaments crossing voids and in superclusters.

2) The values of the environmental densities around multimodal clusters (i.e. those with large number of components, high probabilities to have substructure, and large peculiar velocities of their main galaxies) are higher than those around unimodal clusters.

3) We determined the values of the fourth Minkowski functional and shapefinders, and morphological types for each supercluster hosting clusters from our sample. Of 50 superclusters hosting rich clusters 35 are of spider type and 15 of filament type.

4) Clusters in superclusters of spider morphology have higher probabilities to have substructure, and higher values of the peculiar velocities of their main galaxies than clusters in superclusters of filament morphology.

5) High-luminosity clusters reside in the cores of rich superclusters. Five out of seven high-luminosity clusters belong to superclusters of spider morphology. The most multimodal clusters are preferentially located in spider-type superclusters, while four of seven most unimodal clusters reside in filament-type superclusters.

6) Isolated clusters are poorer and they have smaller maximal number of components and lower maximal (but higher median) values of the peculiar velocities of their main galaxies than supercluster members.

7) High luminosity superclusters have higher values of the environmental densities and peak densities than low luminosity superclusters.

Our study shows the importance of the role of superclusters as high density environment which affects the properties of galaxies and galaxy systems in them (Plionis 2004; Wolf et al. 2005; Haines et al. 2006; Einasto et al. 2007c; Porter et al. 2008; Tempel et al. 2009; Fleenor \& Johnston-Hollitt 2010; Tempel et al. 2011; Einasto et al. 2011e). Earlier studies of galaxy superclusters revealed that while according to their overall properties superclusters can be described with a small number of parameters (Einasto et al. 2011c), the analysis of the morphology and galaxy and group content of the richest superclusters from the $2 \mathrm{dF}$ Galaxy Redshift Survey and from the Sloan Great Wall with SDSS data (Einasto et al. 2008, 2011e) demonstrate a large variety of supercluster morphologies and differences in the distribution of galaxies from various populations, and groups of galaxies in superclusters. Observations have already revealed large differences between galaxy and group content in high- redshift superclusters (Lubin et al. 2009; Schirmer et al. 2011). Such a large variety of observational properties is not yet recovered by simulations (Einasto et al. 2007c, 2011e, and references therein) and not well understood from observations. As a next step we plan to study the properties of a large sample of groups and clusters in superclusters of different morphology to better understand the differences and similarities between them.

Acknowledgements. We thank our referee for very detailed comments, which helped to improve the paper. We are pleased to thank the SDSS Team for the publicly available data releases. Funding for the Sloan Digital Sky Survey (SDSS) and SDSS-II has been provided by the Alfred P. Sloan Foundation, the Participating Institutions, the National Science Foundation, the US Department of Energy, the National Aeronautics and Space Administration, the Japanese Monbukagakusho, and the Max Planck Society, and the Higher Education Funding Council for England. The SDSS Web site is http://www. sdss.org/. The SDSS is managed by the Astrophysical Research Consortium (ARC) for the Participating Institutions. The Participating Institutions are the American Museum of Natural History, Astrophysical Institute Potsdam, University of Basel, University of Cambridge, Case Western Reserve University, The University of Chicago, Drexel University, Fermilab, the Institute for Advanced Study, the Japan Participation Group, The Johns Hopkins University, the Joint Institute for Nuclear Astrophysics, the Kavli Institute for Particle Astrophysics and Cosmology, the Korean Scientist Group, the Chinese Academy of Sciences (LAMOST), Los Alamos National Laboratory, the Max-Planck-Institute for Astronomy (MPIA), the Max-Planck-Institute for Astrophysics (MPA), New Mexico State University, Ohio State University, University of Pittsburgh, University of Portsmouth, Princeton University, the United States Naval Observatory, and the University of Washington. The present study was supported by the Estonian Science Foundation grants Nos. 8005, 7765, 9428, and MJD272, by the Estonian Ministry for Education and Science research project SF0060067s08, and by the European Structural Funds grant for the Centre of Excellence "Dark Matter in (Astro)particle Physics and Cosmology" TK120. This work has also been supported by ICRAnet through a professorship for Jaan Einasto. P.N. was supported by the Academy of Finland, P.H. by Turku University Foundation. V.M. was supported by the Spanish MICINN CONSOLIDER projects ATA2006-14056 and CSD2007-00060, including FEDER contributions, and by the Generalitat Valenciana project of excellence PROMETEO/2009/064.

\section{References}

Aihara, H., Allende Prieto, C., An, D., et al. 2011, ApJS, 193, 29

Andrade-Santos, F., Lima Neto, G. B., \& Laganá, T. F. 2012, ApJ, 746, 139

Araya-Melo, P. A., Reisenegger, A., Meza, A., et al. 2009, MNRAS, 399, 97

Bardelli, S., Zucca, E., Zamorani, G., Moscardini, L., \& Scaramella, R. 2000, MNRAS, 312, 540

Belsole, E., Pratt, G. W., Sauvageot, J., \& Bourdin, H. 2004, A\&A, 415, 821

Bird, C. M., \& Beers, T. C. 1993, AJ, 105, 1596

Blanton, M. R., \& Roweis, S. 2007, AJ, 133, 734

Blanton, M. R., Hogg, D. W., Bahcall, N. A., et al. 2003, ApJ, 592, 819

Boschin, W., Barrena, R., Girardi, M., \& Spolaor, M. 2008, A\&A, 487, 33

Bourdin, H., \& Mazzotta, P. 2008, A\&A, 479, 307

Burgett, W. S., Vick, M. M., Davis, D. S., et al. 2004, MNRAS, 352, 605

Clarke, T. E., Blanton, E. L., \& Sarazin, C. L. 2004, ApJ, 616, 178

Codis, S., Pichon, C., Devriendt, J., et al. 2012, MNRAS, submitted [arXiv: 1201.5794]

Croft, R., Di Matteo, T., Khandai, N., et al. 2011, MNRAS, submitted [arXiv: 1109.4169]

de Lapparent, V., Geller, M. J., \& Huchra, J. P. 1986, ApJ, 302, L1

Einasto, M., Tago, E., Jaaniste, J., Einasto, J., \& Andernach, H. 1997, A\&AS, 123,119

Einasto, M., Einasto, J., Tago, E., Müller, V., \& Andernach, H. 2001, AJ, 122, 2222

Einasto, M., Einasto, J., Müller, V., Heinämäki, P., \& Tucker, D. L. 2003a, A\&A, 401,851

Einasto, M., Jaaniste, J., Einasto, J., et al. 2003b, A\&A, 405, 821

Einasto, M., Suhhonenko, I., Heinämäki, P., Einasto, J., \& Saar, E. 2005, A\&A, 436, 17

Einasto, J., Einasto, M., Saar, E., et al. 2007a, A\&A, 462, 397

Einasto, M., Einasto, J., Tago, E., et al. 2007b, A\&A, 464, 815

Einasto, M., Saar, E., Liivamägi, L. J., et al. 2007c, A\&A, 476, 697

Einasto, M., Saar, E., Martínez, V. J., et al. 2008, ApJ, 685, 83

Einasto, M., Tago, E., Saar, E., et al. 2010, A\&A, 522, A92

Einasto, J., Hütsi, G., Saar, E., et al. 2011a, A\&A, 531, A75

Einasto, J., Suhhonenko, I., Hütsi, G., et al. 2011b, A\&A, 534, A128

Einasto, M., Liivamägi, L. J., Saar, E., et al. 2011c, A\&A, 535, A36

Einasto, M., Liivamägi, L. J., Tago, E., et al. 2011d, A\&A, 532, A5

Einasto, M., Liivamägi, L. J., Tempel, E., et al. 2011e, ApJ, 736, 51 
Einasto, M., Vennik, J., Nurmi, P., et al. 2012, A\&A, 540, A123 (E12)

Espino-Briones, N., Plionis, M., \& Ragone-Figueroa, C. 2007, ApJ, 666, L5

Fakhouri, O., Ma, C.-P., \& Boylan-Kolchin, M. 2010, MNRAS, 406, 2267

Fleenor, M. C., \& Johnston-Hollitt, M. 2010, in ASP Conf. Ser. 423, ed.

B. Smith, J. Higdon, S. Higdon, \& N. Bastian, 81

Flin, P., \& Krywult, J. 2006, A\&A, 450, 9

Fraley, C., \& Raftery, A. E. 2006, Technical Report, Dep. of Statistics, University of Washington, 504, 1

Gastaldello, F., Ettori, S., Balestra, I., et al. 2010, A\&A, 522, A34

Hahn, O., Porciani, C., Carollo, C. M., \& Dekel, A. 2007, MNRAS, 375, 489

Haines, C. P., Merluzzi, P., Mercurio, A., et al. 2006, MNRAS, 371, 55

Hoffman, Y., Lahav, O., Yepes, G., \& Dover, Y. 2007, J. Cosmol. Astropart. Phys., 10, 16

Hou, A., Parker, L. C., Wilman, D. J., et al. 2012, MNRAS, 421, 3594

Huchra, J. P., \& Geller, M. J. 1982, ApJ, 257, 423

Ihaka, R., \& Gentleman, R. 1996, J. Comp. Graph. Stat., 5, 299

Jõeveer, M., Einasto, J., \& Tago, E. 1978, MNRAS, 185, 357

Jeeson-Daniel, A., Dalla Vecchia, C., Haas, M. R., \& Schaye, J. 2011, MNRAS, 415, L69

Knebe, A., \& Müller, V. 2000, A\&A, 354, 761

Kolokotronis, V., Basilakos, S., \& Plionis, M. 2002, MNRAS, 331, 1020

Kopylova, F. G., \& Kopylov, A. I. 2007, Astron. Lett., 33, 211

Kopylova, F. G., \& Kopylov, A. I. 2009, Astrophys. Bull., 64, 1

Liivamägi, L. J., Tempel, E., \& Saar, E. 2012, A\&A, 539, A80

Lim, S., \& Lee, J. 2012 [arXiv: 1201 . 1382]

Loeb, A. 2002, Phys. Rev. D, 65, 047301

Loeb, A. 2008 [arXiv:0804.2258]

Lubin, L. M., Gal, R. R., Lemaux, B. C., Kocevski, D. D., \& Squires, G. K. 2009, AJ, 137, 4867

Markevitch, M., Ponman, T. J., Nulsen, P. E. J., et al. 2000, ApJ, 541, 542

Martínez, V. J., \& Saar, E. 2002, Statistics of the Galaxy Distribution (Boca Raton: Chapman \& Hall/CRC)

Martínez, V. J., Arnalte-Mur, P., Saar, E., et al. 2009, ApJ, 696, L93

McIntosh, D. H., Guo, Y., Hertzberg, J., et al. 2008, MNRAS, 388, 1537

Mo, H. J., \& White, S. D. M. 2002, MNRAS, 336, 112

Noh, Y., \& Cohn, J. D. 2011, MNRAS, 413, 301

Oort, J. H. 1983, ARA\&A, 21, 373

Owers, M. S., Nulsen, P. E. J., Couch, W. J., \& Markevitch, M. 2009, ApJ, 704, 1349

Padilla-Torres, C. P., Gutiérrez, C. M., Rebolo, R., Génova-Santos, R., \& Rubiño-Martin, J. A. 2009, MNRAS, 396, 53
Pan, Z., Yuan, Q., Kong, X., et al. 2012, MNRAS, 2432

Park, C., Choi, Y., Vogeley, M. S., Gott, III, J. R., \& Blanton, M. R. 2007, ApJ, 658,898

Pinkney, J., Roettiger, K., Burns, J. O., \& Bird, C. M. 1996, ApJS, 104, 1

Plionis, M. 2004, in Outskirts of Galaxy Clusters: Intense Life in the Suburbs, ed. A. Diaferio, IAU Colloq., 195, 19

Plionis, M., \& Basilakos, S. 2002, MNRAS, 329, L47

Pompei, E., \& Iovino, A. 2012, A\&A, 539, A106

Porter, S. C., Raychaudhury, S., Pimbblet, K. A., \& Drinkwater, M. J. 2008, MNRAS, 388, 1152

Power, C., Knebe, A., \& Knollmann, S. R. 2012, MNRAS, 419, 1576

Pratt, G. W., \& Arnaud, M. 2003, A\&A, 408, 1

Ragone-Figueroa, C., \& Plionis, M. 2007, MNRAS, 377, 1785

Richstone, D., Loeb, A., \& Turner, E. L. 1992, ApJ, 393, 477

Rose, J. A., Gaba, A. E., Christiansen, W. A., et al. 2002, AJ, 123, 1216

Saar, E. 2009, in Data Analysis in Cosmology, ed. V. J. Martínez, E. Saar, E. Martínez-Gonzalez, \& M.-J. Pons-Bordería (Berlin: Springer-Verlag), 523

Saar, E., Martínez, V. J., Starck, J., \& Donoho, D. L. 2007, MNRAS, 374, 1030

Sahni, V., Sathyaprakash, B. S., \& Shandarin, S. F. 1998, ApJ, 495, L5

Schirmer, M., Hildebrandt, H., Kuijken, K., \& Erben, T. 2011, A\&A, 532, A57

Shandarin, S. F., Sheth, J. V., \& Sahni, V. 2004, MNRAS, 353, 162

Sheth, R. K., \& Diaferio, A. 2011, MNRAS, 417, 2938

Silverman, B. W. 1986, Density Estimation for Statistics and Data Analysis (Boca Raton: Chapman \& Hall, CRC Press)

Skibba, R. A., \& Macciò, A. V. 2011, MNRAS, 416, 2388

Small, T. A., Ma, C., Sargent, W. L. W., \& Hamilton, D. 1998, ApJ, 492, 45

Smith, G. P., \& Taylor, J. E. 2008, ApJ, 682, L73

Solanes, J. M., Salvador-Solé, E., \& González-Casado, G. 1999, A\&A, 343, 733

Suhhonenko, I., Einasto, J., Liivamägi, L. J., et al. 2011, A\&A, 531, A149

Tago, E., Einasto, J., Saar, E., et al. 2008, A\&A, 479, 927

Tago, E., Saar, E., Tempel, E., et al. 2010, A\&A, 514, A102

Tempel, E., Einasto, J., Einasto, M., Saar, E., \& Tago, E. 2009, A\&A, 495, 37

Tempel, E., Saar, E., Liivamägi, L. J., et al. 2011, A\&A, 529, A53

Tempel, E., Tago, E., \& Liivamägi, L. J. 2012, A\&A, 540, A106

Turner, E. L., \& Gott, III, J. R. 1976, ApJS, 32, 409

Udomprasert, P. S. 2004, Ph.D. Thesis, California Institute of Technology, United States - California

Wang, H., Mo, H. J., Jing, Y. P., Yang, X., \& Wang, Y. 2011, MNRAS, 413, 1973

White, M., Cohn, J. D., \& Smit, R. 2010, MNRAS, 408, 1818

Wolf, C., Gray, M. E., \& Meisenheimer, K. 2005, A\&A, 443, 435

Zeldovich, I. B., Einasto, J., \& Shandarin, S. F. 1982, Nature, 300, 407 
Table 5. Data on clusters.

\begin{tabular}{|c|c|c|c|c|c|c|c|c|c|c|c|c|}
\hline $\begin{array}{l}\text { (1) } \\
\text { ID }\end{array}$ & $\begin{array}{l}(2) \\
N_{\text {gal }}\end{array}$ & $\begin{array}{c}(3) \\
L_{\mathrm{tot}} \\
10^{10} h^{-2} L_{\odot} \\
\end{array}$ & $\begin{array}{c}(4) \\
\sigma \\
\mathrm{km} \mathrm{s}^{-1} \\
\end{array}$ & $\begin{array}{c}(5) \\
r_{\mathrm{vir}} \\
h^{-1} \mathrm{Mpc} \\
\end{array}$ & $\begin{array}{c}(6) \\
V_{\mathrm{pec}} \\
\mathrm{km} \mathrm{s}^{-1} \\
\end{array}$ & $\begin{array}{c}(7) \\
N_{\text {comp }}\end{array}$ & $\begin{array}{l}(8) \\
p_{\Delta}\end{array}$ & $\begin{array}{l}(9) \\
D 4\end{array}$ & $\begin{array}{c}(10) \\
D 8\end{array}$ & $\begin{array}{l}(11) \\
D 16\end{array}$ & $\begin{array}{l}(12) \\
I D_{\mathrm{scl}}\end{array}$ & $\begin{array}{c}(13) \\
\text { Abell ID }\end{array}$ \\
\hline 18 & 87 & 110 & 513 & 0.73 & 387 & 2 & $<10^{-4}$ & 25.7 & 6.8 & 2.7 & 211 & - \\
\hline 323 & 67 & 73 & 276 & 0.67 & 237 & 3 & 0.180 & 18.2 & 4.3 & 1.5 & 0 & - \\
\hline 608 & 60 & 132 & 532 & 0.66 & -228 & 1 & 0.884 & 33.4 & 7.6 & 2.7 & 569 & A2175 \\
\hline 748 & 79 & 93 & 748 & 0.43 & 613 & 1 & 0.007 & 37.1 & 8.2 & 2.8 & 319 & A1066 \\
\hline 793 & 122 & 63 & 515 & 0.56 & -384 & 3 & $<10^{-4}$ & 19.4 & 4.4 & 2.1 & 0 & A2107 \\
\hline 880 & 57 & 101 & 411 & 0.84 & 400 & 3 & 0.002 & 26.6 & 6.9 & 2.8 & 27 & - \\
\hline 914 & 119 & 227 & 657 & 0.83 & -704 & 5 & $<10^{-4}$ & 49.5 & 13.2 & 4.0 & 27 & A 1750 \\
\hline 1469 & 56 & 99 & 418 & 0.69 & -342 & 2 & 0.198 & 32.3 & 9.1 & 3.2 & 126 & A933 \\
\hline 1573 & 57 & 35 & 744 & 0.25 & 1228 & 3 & 0.334 & -999 & -999 & -999 & 0 & - \\
\hline 1944 & 60 & 47 & 440 & 0.46 & -122 & 3 & 0.020 & 16.4 & 4.2 & 1.7 & 0 & - \\
\hline 2067 & 62 & 66 & 574 & 0.44 & -788 & 2 & 0.007 & 21.6 & 5.0 & 1.5 & 0 & - \\
\hline 3714 & 82 & 54 & 344 & 0.60 & -6 & 2 & $<10^{-4}$ & 19.4 & 5.2 & 1.5 & 0 & A1139 \\
\hline 4122 & 88 & 68 & 963 & 0.49 & -1091 & 3 & $<10^{-4}$ & 19.8 & 5.6 & 2.7 & 515 & A1291 \\
\hline 4713 & 80 & 80 & 637 & 0.43 & -78 & 3 & 0.003 & 25.6 & 7.6 & 2.9 & 515 & A1377 \\
\hline 4744 & 71 & 88 & 492 & 0.62 & 147 & 4 & 0.001 & 25.5 & 6.7 & 2.6 & 499 & A1238 \\
\hline 4992 & 68 & 157 & 619 & 0.65 & 303 & 2 & 0.048 & 48.9 & 10.1 & 3.3 & 103 & A2048 \\
\hline 5217 & 89 & 94 & 577 & 0.61 & -19 & 1 & 0.216 & 27.3 & 8.0 & 3.2 & 211 & A1436 \\
\hline 7102 & 54 & 33 & 465 & 0.46 & 326 & 2 & 0.022 & 14.1 & 4.8 & 2.2 & 1238 & - \\
\hline 7932 & 50 & 99 & 413 & 0.55 & -111 & 2 & 0.089 & 29.8 & 8.5 & 2.9 & 124 & A724 \\
\hline 9029 & 78 & 99 & 312 & 0.72 & -341 & 2 & 0.094 & 28.4 & 7.4 & 2.1 & 491 & A865 \\
\hline 9350 & 89 & 55 & 480 & 0.65 & -129 & 4 & 0.020 & 18.5 & 7.3 & 2.7 & 352 & A2055, A2063 \\
\hline 9985 & 58 & 41 & 384 & 0.43 & 57 & 2 & 0.167 & 18.6 & 6.4 & 2.4 & 782 & - \\
\hline 10438 & 65 & 45 & 395 & 0.46 & -161 & 2 & 0.008 & 15.4 & 4.9 & 2.1 & 782 & - \\
\hline 11015 & 52 & 45 & 303 & 0.46 & 140 & 8 & 0.037 & 17.9 & 4.5 & 1.5 & 0 & - \\
\hline 11474 & 51 & 36 & 306 & 0.49 & -267 & 5 & 0.001 & 11.5 & 3.1 & 1.1 & 0 & - \\
\hline 11683 & 54 & 52 & 342 & 0.46 & -21 & 1 & 0.064 & 17.3 & 3.5 & 1.8 & 0 & A1507 \\
\hline 12508 & 93 & 90 & 385 & 0.77 & -78 & 2 & $<10^{-4}$ & 21.2 & 6.0 & 1.7 & 850 & A2169 \\
\hline 12540 & 103 & 107 & 764 & 0.52 & 477 & 2 & 0.345 & 35.3 & 9.8 & 3.4 & 143 & A1767 \\
\hline 13216 & 57 & 48 & 400 & 0.54 & 261 & 3 & $<10^{-4}$ & 16.3 & 3.6 & 1.3 & 0 & - \\
\hline 13347 & 50 & 64 & 479 & 0.48 & 369 & 2 & 0.007 & 19.8 & 4.9 & 1.5 & 0 & A1003 \\
\hline 13408 & 58 & 62 & 282 & 0.73 & 114 & 2 & 0.752 & 18.8 & 6.0 & 1.7 & 849 & A2149 \\
\hline 16094 & 71 & 113 & 735 & 0.50 & -47 & 3 & 0.021 & 37.1 & 8.8 & 2.4 & 218 & A1691 \\
\hline 16309 & 69 & 151 & 878 & 0.46 & 84 & 4 & 0.004 & 43.4 & 9.1 & 2.6 & 59 & A2244 \\
\hline 16350 & 65 & 135 & 859 & 0.36 & -626 & 2 & $<10^{-4}$ & 43.4 & 8.6 & 2.9 & 233 & A2245 \\
\hline 17210 & 72 & 120 & 872 & 0.47 & -1308 & 2 & 0.007 & 36.0 & 8.4 & 2.7 & 233 & A2249 \\
\hline 18029 & 53 & 37 & 597 & 0.41 & -625 & 3 & $<10^{-4}$ & 12.4 & 2.8 & 1.1 & 0 & A1781 \\
\hline 18048 & 78 & 121 & 596 & 0.61 & 28 & 2 & 0.010 & 39.7 & 12.0 & 4.2 & 54 & - \\
\hline 20159 & 52 & 59 & 517 & 0.36 & -300 & 2 & 0.011 & 20.6 & 4.1 & 1.3 & 0 & A1026, A1035 \\
\hline 20419 & 58 & 44 & 424 & 0.59 & 201 & 3 & 0.004 & 15.1 & 3.6 & 1.7 & 0 & A1749 \\
\hline 20514 & 56 & 33 & 317 & 0.42 & -390 & 4 & 0.436 & 12.5 & 3.9 & 1.7 & 0 & - \\
\hline 21573 & 50 & 60 & 364 & 0.49 & -32 & 2 & 0.488 & 22.4 & 6.6 & 2.3 & 797 & - \\
\hline 22572 & 77 & 75 & 533 & 0.43 & -33 & 2 & $<10^{-4}$ & 21.1 & 4.5 & 1.5 & 0 & A1169 \\
\hline 23374 & 114 & 100 & 662 & 0.72 & 1226 & 2 & 0.001 & 27.6 & 8.3 & 3.4 & 219 & A1795, A1818 \\
\hline 23524 & 50 & 63 & 304 & 0.67 & -68 & 2 & 0.166 & 19.8 & 7.5 & 2.9 & 220 & - \\
\hline 24554 & 50 & 83 & 658 & 0.58 & 107 & 3 & $<10^{-4}$ & 27.9 & 10.1 & 4.4 & 27 & A1620 \\
\hline 24604 & 50 & 59 & 857 & 0.50 & -256 & 2 & 0.001 & 21.2 & 6.8 & 3.0 & 220 & A1831 \\
\hline 24829 & 77 & 126 & 534 & 0.66 & -107 & 3 & 0.003 & 36.8 & 11.5 & 4.3 & 27 & A1663 \\
\hline 25078 & 51 & 87 & 498 & 0.61 & -120 & 1 & 0.270 & 30.0 & 11.8 & 4.9 & 27 & A1650 \\
\hline 28272 & 51 & 40 & 355 & 0.50 & 464 & 1 & 0.032 & 13.0 & 4.8 & 1.3 & 868 & - \\
\hline 28387 & 88 & 121 & 481 & 0.66 & -167 & 4 & $<10^{-4}$ & 28.5 & 8.4 & 3.4 & 211 & - \\
\hline 28508 & 58 & 74 & 494 & 0.40 & 478 & 1 & 0.482 & 24.8 & 8.0 & 3.3 & 211 & A1270 \\
\hline 28986 & 66 & 73 & 398 & 0.69 & -205 & 5 & 0.002 & 19.7 & 6.4 & 2.8 & 360 & A2092 \\
\hline 29348 & 75 & 119 & 418 & 0.69 & 9 & 4 & 0.001 & 33.7 & 8.7 & 3.1 & 99 & - \\
\hline 29350 & 55 & 92 & 334 & 0.64 & -309 & 2 & 0.325 & 29.9 & 8.0 & 3.1 & 99 & - \\
\hline 29587 & 207 & 365 & 740 & 0.87 & 334 & 3 & $<10^{-4}$ & 79.3 & 21.3 & 5.8 & 1 & A2142 \\
\hline 29744 & 53 & 96 & 396 & 0.76 & -557 & 3 & 0.004 & 24.0 & 5.9 & 2.1 & 870 & A1939 \\
\hline 30391 & 68 & 93 & 271 & 0.73 & 279 & 3 & $<10^{-4}$ & 20.4 & 4.6 & 1.4 & 0 & - \\
\hline 32006 & 71 & 72 & 420 & 0.53 & 11 & 2 & $<10^{-4}$ & 19.9 & 5.6 & 2.7 & 211 & A1396, A1400 \\
\hline 32663 & 51 & 61 & 435 & 0.73 & 96 & 1 & 0.008 & 19.3 & 4.8 & 2.0 & 0 & - \\
\hline 32909 & 79 & 69 & 560 & 0.56 & -223 & 3 & 0.006 & 22.8 & 5.3 & 1.8 & 1244 & A2022 \\
\hline 32976 & 64 & 72 & 510 & 0.68 & 157 & 2 & 0.053 & 21.7 & 4.8 & 1.4 & 0 & A1808 \\
\hline 33082 & 77 & 79 & 382 & 0.52 & -137 & 2 & 0.120 & 25.8 & 6.9 & 2.9 & 211 & A1383, A1396, A1400 \\
\hline 33739 & 79 & 65 & 517 & 0.49 & -217 & 4 & 0.003 & 21.5 & 5.0 & 2.0 & 0 & A1890 \\
\hline
\end{tabular}


Table 5. continued.

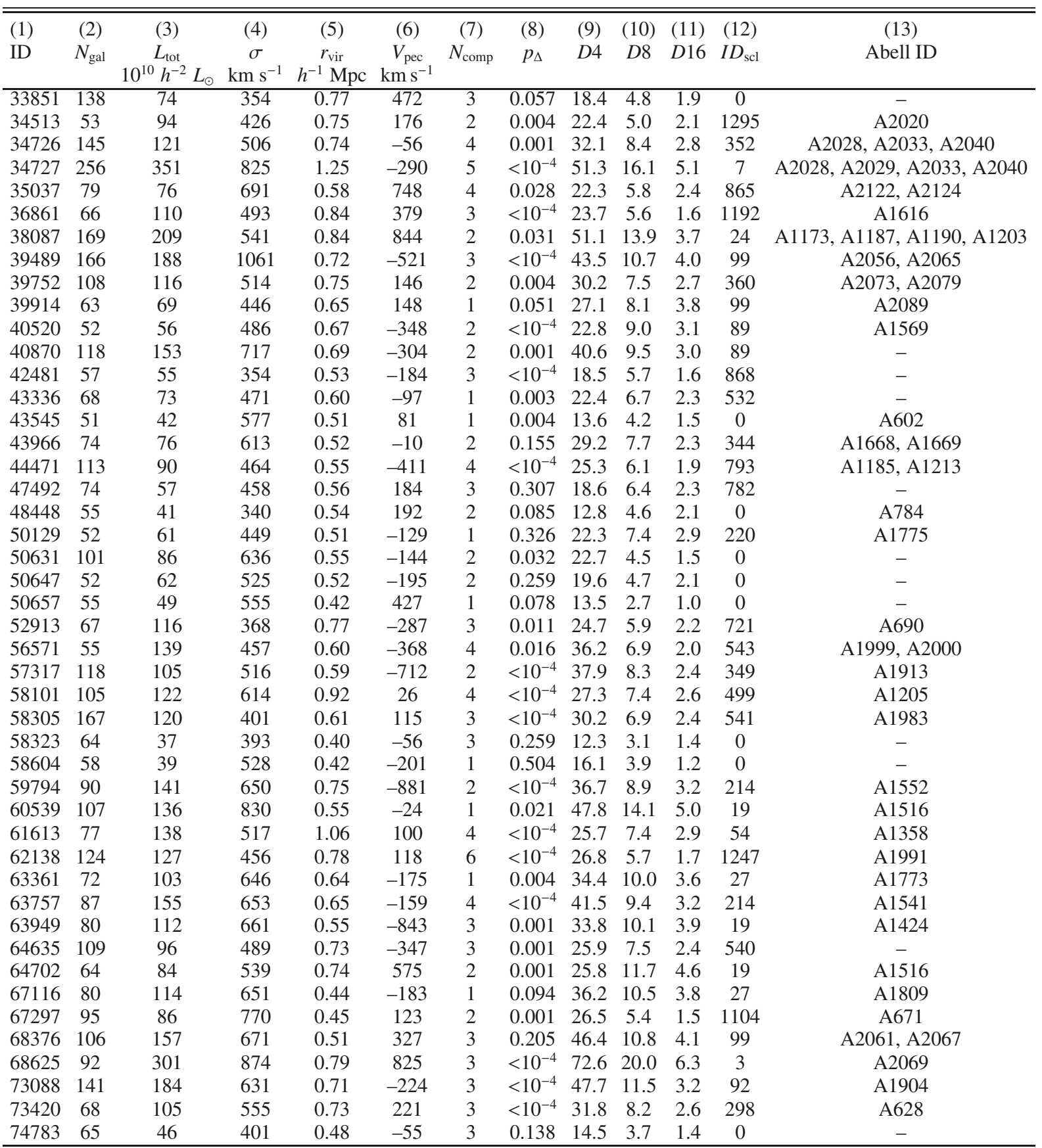

Notes. Columns are as follows: 1: ID of a cluster; 2 : the number of galaxies in the cluster, $N_{\mathrm{gal}} ; 3$ : total luminosity of the cluster; 4 : rms velocity of the cluster; 5: virial radius of the cluster; 6: peculiar velocity of the main galaxy; 7: the number of components in the cluster, $N_{\text {comp }} ; 8: p$-value of the $\Delta$ test; 9-11: environmental density around the cluster, at smoothing lengths 4, 8, and $16 h^{-1} \mathrm{Mpc}$ (in units of the mean density; -999 denotes cluster at the edge of a survey where the density cannot be calculated); 12: ID of the supercluster where the cluster resides; 13: Abell ID of the cluster. 
Table 6. Data on superclusters.

\begin{tabular}{|c|c|c|c|c|c|c|c|c|c|c|c|c|}
\hline (1) & (2) & (3) & (4) & (5) & (6) & (7) & (8) & (9) & $(10)$ & (11) & (12) & (13) \\
\hline ID(long) & ID(DR8) & ID(DR7) & $N_{\text {gal }}$ & $\begin{array}{c}\text { Dist. } \\
h^{-1} \mathrm{Mpc}\end{array}$ & $\begin{array}{c}L_{\mathrm{tot}} \\
10^{10} h^{-2} L_{\odot}\end{array}$ & $D_{\text {peak }}$ & $V_{3}$ & $K_{1}$ & $K_{2}$ & $K_{1} / K_{2}$ & Type & $\mathrm{ID}(\mathrm{E} 01)$ \\
\hline $239+027+0091$ & 1 & 1 & 1041 & 264 & 1809 & 22.2 & 2.5 & 0.07 & 0.17 & 0.41 & $\bar{F}$ & 162 \\
\hline $231+030+0117$ & 3 & 5 & 1191 & 336 & 3694 & 20.6 & 8.5 & 0.12 & 0.17 & 0.74 & $\mathrm{~S}$ & 157 \\
\hline $227+006+0078$ & 7 & 11 & 1217 & 233 & 1675 & 17.0 & 3.0 & 0.04 & 0.08 & 0.56 & $\mathrm{~S}$ & 154 \\
\hline $184+003+0077$ & 19 & 24 & 2060 & 231 & 2919 & 15.0 & 9.0 & 0.11 & 0.34 & 0.33 & $S$ & 111 \\
\hline $167+040+0078$ & 24 & 38 & 580 & 225 & 751 & 14.6 & 2.0 & 0.02 & 0.03 & 0.86 & $\mathrm{~S}$ & 95 \\
\hline $202-001+0084$ & 27 & 61 & 3222 & 256 & 5163 & 14.0 & 14.5 & 0.12 & 0.46 & 0.26 & $\mathrm{~F}$ & 126 \\
\hline $173+014+0082$ & 54 & 55 & 1341 & 241 & 2064 & 12.4 & 5.5 & 0.09 & 0.16 & 0.56 & $\mathrm{~S}$ & 111 \\
\hline $250+027+0102$ & 59 & 64 & 656 & 301 & 1563 & 12.8 & 5.0 & 0.08 & 0.22 & 0.36 & $\mathrm{~F}$ & 164 \\
\hline $189+017+0071$ & 89 & 136 & 515 & 212 & 610 & 11.5 & 2.0 & 0.03 & 0.01 & 1.98 & $\mathrm{~S}$ & 271 \\
\hline $215+048+0071$ & 92 & 87 & 527 & 213 & 690 & 11.8 & 2.5 & 0.05 & 0.12 & 0.40 & $\mathrm{~S}$ & - \\
\hline $230+027+0070$ & 99 & 94 & 2047 & 215 & 2874 & 11.5 & 10.0 & 0.11 & 0.43 & 0.26 & $\mathrm{~S}$ & 158 \\
\hline $229+006+0102$ & 103 & 152 & 425 & 302 & 1004 & 11.0 & 3.0 & 0.05 & 0.10 & 0.50 & $\mathrm{~F}$ & 160 \\
\hline $135+038+0094$ & 124 & 195 & 273 & 281 & 548 & 10.5 & 2.0 & 0.02 & 0.03 & 0.62 & $\mathrm{~S}$ & 249 \\
\hline $152-000+0096$ & 126 & 198 & 495 & 285 & 1001 & 10.1 & 4.0 & 0.05 & 0.13 & 0.37 & $\mathrm{~F}$ & 82 \\
\hline $203+059+0072$ & 143 & 228 & 668 & 211 & 753 & 10.1 & 2.0 & 0.04 & 0.03 & 1.27 & $\mathrm{~F}$ & 133 \\
\hline $172+054+0071$ & 211 & 336 & 1439 & 207 & 1618 & 9.2 & 7.0 & 0.10 & 0.28 & 0.36 & $\mathrm{~F}$ & 109 \\
\hline $187+008+0090$ & 214 & 223 & 735 & 268 & 1218 & 9.7 & 6.0 & 0.08 & 0.30 & 0.28 & $\mathrm{~S}$ & 111 \\
\hline $197+039+0073$ & 218 & 344 & 272 & 215 & 337 & 9.1 & 1.0 & 0.00 & -0.00 & -20.02 & $\mathrm{~S}$ & 274 \\
\hline $207+026+0067$ & 219 & 349 & 985 & 187 & 1007 & 9.2 & 4.0 & 0.07 & 0.11 & 0.61 & $\mathrm{~S}$ & 138 \\
\hline $207+028+0077$ & 220 & 351 & 603 & 226 & 768 & 9.0 & 4.0 & 0.05 & 0.10 & 0.47 & $\mathrm{~F}$ & 138 \\
\hline $255+033+0086$ & 233 & 376 & 474 & 259 & 790 & 9.0 & 4.0 & 0.04 & 0.04 & 0.96 & $\mathrm{~F}$ & 167 \\
\hline $122+035+0084$ & 298 & 311 & 246 & 246 & 345 & 8.6 & 1.0 & 0.02 & 0.00 & 4.27 & $S$ & 75 \\
\hline $159+004+0069$ & 319 & 503 & 245 & 207 & 296 & 8.3 & 2.0 & 0.02 & 0.02 & 0.97 & S & 91 \\
\hline $195+019+0064$ & 344 & 538 & 264 & 192 & 290 & 8.1 & 1.0 & -0.00 & 0.01 & -0.14 & $\mathrm{~S}$ & 273 \\
\hline $216+016+0051$ & 349 & 548 & 335 & 159 & 284 & 8.5 & 1.0 & 0.01 & 0.01 & 0.97 & $S$ & 143 \\
\hline $227+007+0045$ & 352 & 550 & 519 & 135 & 379 & 8.7 & 1.0 & 0.02 & 0.01 & 1.46 & $\mathrm{~S}$ & 154 \\
\hline $232+029+0066$ & 360 & 362 & 311 & 196 & 330 & 8.4 & 2.0 & 0.00 & -0.01 & -0.54 & $\mathrm{~S}$ & 158 \\
\hline $146+043+0072$ & 491 & 500 & 199 & 217 & 256 & 7.8 & 1.0 & 0.00 & -0.01 & -0.15 & S & - \\
\hline $168+002+0077$ & 499 & 512 & 408 & 228 & 517 & 7.8 & 3.0 & 0.05 & 0.08 & 0.67 & $\mathrm{~F}$ & 91 \\
\hline $176+055+0052$ & 515 & 525 & 457 & 155 & 390 & 7.9 & 3.0 & 0.02 & -0.00 & -11.10 & $S$ & 109 \\
\hline $208+046+0062$ & 532 & 543 & 297 & 189 & 293 & 7.4 & 2.0 & 0.01 & 0.02 & 0.49 & $\mathrm{~F}$ & - \\
\hline $214+002+0053$ & 540 & 549 & 422 & 163 & 358 & 7.9 & 1.5 & 0.02 & 0.05 & 0.49 & S & - \\
\hline $223+016+0045$ & 541 & 849 & 299 & 135 & 214 & 7.2 & 1.0 & 0.01 & -0.01 & -0.88 & S & - \\
\hline $223+054+0098$ & 543 & 552 & 80 & 294 & 186 & 7.0 & 1.0 & 0.01 & -0.04 & -0.40 & S & 147 \\
\hline $245+029+0096$ & 569 & 578 & 198 & 285 & 417 & 7.9 & 2.0 & 0.04 & 0.05 & 0.79 & $S$ & 164 \\
\hline $129+028+0079$ & 721 & 1094 & 94 & 237 & 145 & 6.2 & 1.0 & -0.00 & -0.01 & 0.22 & $S$ & 76 \\
\hline $151+054+0047$ & 782 & 779 & 652 & 139 & 465 & 6.8 & 3.0 & 0.05 & 0.14 & 0.38 & S & - \\
\hline $169+029+0046$ & 793 & 1184 & 211 & 142 & 156 & 6.3 & 1.0 & -0.01 & 0.01 & -1.24 & S & 93 \\
\hline $176+015+0069$ & 797 & 794 & 160 & 205 & 203 & 6.7 & 1.0 & 0.00 & 0.01 & 0.22 & S & - \\
\hline $240+053+0065$ & 849 & 857 & 215 & 194 & 242 & 6.5 & 1.0 & 0.00 & -0.02 & -0.03 & $\mathrm{~F}$ & 162 \\
\hline $244+049+0057$ & 850 & 1258 & 135 & 171 & 128 & 6.2 & 1.0 & -0.01 & -0.02 & 0.63 & S & 162 \\
\hline $234+036+0065$ & 865 & 1278 & 161 & 197 & 170 & 6.0 & 2.0 & 0.02 & -0.01 & -1.68 & S & 158 \\
\hline $246+014+0050$ & 868 & 1283 & 239 & 153 & 201 & 6.4 & 1.5 & -0.00 & -0.00 & 0.98 & $\mathrm{~S}$ & - \\
\hline $219+024+0087$ & 870 & 1284 & 76 & 261 & 143 & 6.1 & 1.0 & 0.00 & -0.01 & -0.14 & $\mathrm{~S}$ & - \\
\hline $127+030+0051$ & 1104 & 1683 & 127 & 150 & 110 & 5.6 & 1.0 & -0.01 & -0.02 & 0.68 & $\mathrm{~S}$ & - \\
\hline $191+054+0085$ & 1192 & 1208 & 79 & 248 & 134 & 5.9 & 1.0 & -0.01 & -0.00 & 1.41 & S & - \\
\hline $220+010+0051$ & 1238 & 1255 & 133 & 156 & 97 & 5.5 & 1.0 & 0.00 & -0.00 & -0.28 & $\mathrm{~F}$ & - \\
\hline $226+028+0057$ & 1244 & 1262 & 101 & 174 & 97 & 5.4 & 1.0 & -0.01 & -0.02 & 0.76 & $\mathrm{~S}$ & 152 \\
\hline $223+018+0059$ & 1247 & 1265 & 147 & 176 & 149 & 5.9 & 1.0 & -0.00 & -0.03 & 0.28 & $\mathrm{~F}$ & - \\
\hline $226+007+0092$ & 1295 & 1311 & 110 & 272 & 200 & 5.4 & 2.0 & 0.02 & 0.04 & 0.44 & $\mathrm{~F}$ & - \\
\hline
\end{tabular}

Notes. The columns are as follows: 1: long ID of a supercluster AAA+BBB+ZZZZ, where AAA is RA, +/-BBB is Dec (in degrees), and ZZZZ is 1000z; 2: ID of a supercluster from the SDSS DR8 superclusters catalogue; 3: ID of a supercluster from the SDSS DR7 superclusters catalogue (Liivamägi et al. 2012); 4: the number of galaxies in the supercluster, $N_{\text {gal }}$; 5 : the distance of a supercluster; 6 : the total weighted luminosity of galaxies in the supercluster, $L_{\text {tot }}$; 7: the density at the density maximum, $d_{\text {peak }}$, in units of mean density; 8: the maximum value of the fourth Minkowski functional $V_{3}$ (clumpiness) for the supercluster; 9-11: the shapefinders $K_{1}$ (planarity) and $K_{2}$ (filamentarity), and the ratio of the shapefinders $K_{1} / K_{2}$ for the full supercluster; 12: morphological type of a supercluster 13: ID(E01): the supercluster ID in the catalogue by Einasto et al. (2001). SCl 160 - the Hercules supercluster, SCl 111 and SCl 126 - members of the Sloan Great Wall, SCl 158 - the Corona Borealis supercluster, SCl 138 - the Bootes supercluster, SCl 336 - the Ursa Major supercluster. 


\section{Appendix A: Multimodality of clusters}

We employ two 3D methods to search for substructure in clusters. With Mclust package (Fraley \& Raftery 2006) from $R$, an open-source free statistical environment developed under the GNU GPL (Ihaka \& Gentleman 1996, http://www . r-project.org) we search for an optimal model for the clustering of the data among models with varying shape, orientation and volume, under assumption that the multivariate sample is a random sample from a mixture of multivariate normal distributions. Mclust finds the optimal number of components and calculates for every galaxy the probabilities to belong to any of the components which are used to calculate the uncertainties for galaxies to belong to a component. The mean uncertainty for the full sample is used as a statistical estimate of the reliability of the results. The calculations in E12 showed that uncertainties are small, their values can be found in online tables of E12. We tested how the possible errors in the line-of-sight positions of galaxies affect the results of Mclust, shifting randomly the peculiar velocities of galaxies 1000 times and searching each time for the components with Mclust. The random shifts were chosen from a Gaussian distribution with the dispersion equal to the sample velocity dispersion of galaxies in a cluster. The number of the components found by Mclust remained unchanged, demonstrating that the results of Mclust are not sensitive to such errors.

The Dressler-Shectman (DS) test searches for deviations of the local velocity mean and dispersion from the cluster mean values. The algorithm starts by calculating the mean velocity $\left(v_{\text {local }}\right)$ and the velocity dispersion $\left(\sigma_{\text {local }}\right)$ for each galaxy of the cluster, using its $n$ nearest neighbours. These values of local kinematics are compared with the mean velocity $\left(v_{\mathrm{c}}\right)$ and the velocity dispersion $\left(\sigma_{\mathrm{c}}\right)$ determined for the entire cluster of $N_{\text {gal }}$ galaxies. The differences between the local and global kinematics are quantified by

$\delta_{i}^{2}=(n+1) / \sigma_{\mathrm{c}}^{2}\left[\left(v_{\text {local }}-v_{\mathrm{c}}\right)^{2}+\left(\sigma_{\text {local }}-\sigma_{\mathrm{c}}^{2}\right)\right]$.

The cumulative deviation $\Delta=\Sigma \delta_{i}$ is used as a statistic for quantifying (the significance of) the substructure. The results of the DS-test depend on the number of local galaxies $n$. We use $n=\sqrt{N}_{\text {gal }}$, as suggested by Pinkney et al. (1996). The $\Delta$ statistic for each cluster should be calibrated by Monte Carlo simulations. In Monte Carlo models the velocities of galaxies are randomly shuffled among the positions. We ran 25000 models for each cluster and calculated every time $\Delta_{\text {sim. }}$. The significance of having substructure (the $p_{\Delta}$-value) can be quantified by the ratio $N\left(\Delta_{\text {sim }}>\Delta_{\text {obs }}\right) / N_{\text {sim }}$ - the ratio of the number of simulations in which the value of $\Delta$ is larger than the observed value, and the total number of simulations. The smaller the $p_{\Delta}$-value, the larger is the probability of substructure.

\section{Appendix B: Luminosity density field and superclusters}

To calculate the luminosity density field, we calculate the luminosities of groups first. In flux-limited samples, galaxies outside the observational window remain unobserved. To take into account the luminosities of the galaxies that lie outside the sample limits also we multiply the observed galaxy luminosities by the weight $W_{\mathrm{d}}$. The distance-dependent weight factor $W_{\mathrm{d}}$ was calculated as

$W_{d}=\frac{\int_{0}^{\infty} \operatorname{Ln}(L) \mathrm{d} L}{\int_{L_{1}}^{L_{2}} \operatorname{Ln}(L) \mathrm{d} L}$, where $L_{1,2}=L_{\odot} 10^{0.4\left(M_{\odot}-M_{1,2}\right)}$ are the luminosity limits of the observational window at a distance $d$, corresponding to the absolute magnitude limits of the window $M_{1}$ and $M_{2}$; we took $M_{\odot}=4.64 \mathrm{mag}$ in the $r$-band (Blanton \& Roweis 2007). Due to their peculiar velocities, the distances of galaxies are somewhat uncertain; if the galaxy belongs to a group, we use the group distance to determine the weight factor. Details of the calculations of weights are given also in Tempel et al. (2011) and in Einasto et al. (2011c).

To calculate a luminosity density field, we convert the spatial positions of galaxies $\boldsymbol{r}_{i}$ and their luminosities $L_{i}$ into spatial (luminosity) densities using kernel densities (Silverman 1986):

$\rho(\boldsymbol{r})=\sum_{i} K\left(\boldsymbol{r}-\boldsymbol{r}_{i} ; a\right) L_{i}$

where the sum is over all galaxies, and $K(\boldsymbol{r} ; a)$ is a kernel function of a width $a$. Good kernels for calculating densities on a spatial grid are generated by box splines $B_{J}$. Box splines are local and they are interpolating on a grid:

$\sum_{i} B_{J}(x-i)=1$,

for any $x$ and a small number of indices that give non-zero values for $B_{J}(x)$. We use the popular $B_{3}$ spline function:

$$
\begin{aligned}
B_{3}(x)= & \left(|x-2|^{3}-4|x-1|^{3}+6|x|^{3}\right. \\
& \left.-4|x+1|^{3}+|x+2|^{3}\right) / 12 .
\end{aligned}
$$

The (one-dimensional) $B_{3}$ box spline kernel $K_{B}^{(1)}$ of the width $a$ is defined as

$K_{B}^{(1)}(x ; a, \delta)=B_{3}(x / a)(\delta / a)$,

where $\delta$ is the grid step. This kernel differs from zero only in the interval $x \in[-2 a, 2 a]$. It is close to a Gaussian with $\sigma=$ 0.6 in the region $x \in[-a, a]$, so its effective width is $2 a$ (see, e.g., Saar 2009). The kernel preserves the interpolation property exactly for all values of $a$ and $\delta$, where the ratio $a / \delta$ is an integer. (This kernel can be used also if this ratio is not an integer, and $a \gg \delta$; the kernel sums to 1 in this case, too, with a very small error.) This means that if we apply this kernel to $N$ points on a one-dimensional grid, the sum of the densities over the grid is exactly $N$.

The three-dimensional kernel $K_{B}^{(3)}$ is given by the direct product of three one-dimensional kernels:

$K_{B}^{(3)}(\boldsymbol{r} ; a, \delta) \equiv K_{3}^{(1)}(x ; a, \delta) K_{3}^{(1)}(y ; a, \delta) K_{3}^{(1)}(z ; a, \delta)$,

where $\boldsymbol{r} \equiv\{x, y, z\}$. Although this is a direct product, it is isotropic to a good degree (Saar 2009).

The densities were calculated on a cartesian grid based on the SDSS $\eta, \lambda$ coordinate system. The grid coordinates are calculated according to Eq. (3). We used an $1 h^{-1} \mathrm{Mpc}$ step grid and chose the kernel width $a=8 h^{-1} \mathrm{Mpc}$. This kernel differs from zero within the radius $16 h^{-1} \mathrm{Mpc}$, but significantly so only inside the $8 h^{-1} \mathrm{Mpc}$ radius. As a lower limit for the volume of superclusters we used the value $(a / 2) h^{-1} \mathrm{Mpc}^{3}$ (64 grid cells). We also used density field with the kernel widths $a=4 h^{-1} \mathrm{Mpc}$, $a=8 h^{-1} \mathrm{Mpc}$, and $a=16 h^{-1} \mathrm{Mpc}$ to characterise the environmental density around clusters. Before extracting superclusters we apply the DR7 mask constructed by Arnalte-Mur (Martínez et al. 2009; Liivamägi et al. 2012) to the density field and convert densities into units of mean density. The mean density is defined as the average over all pixel values inside the mask. The mask is designed to follow the edges of the survey and the galaxy distribution inside the mask is assumed to be homogeneous. 


\section{Appendix C: Minkowski functionals and shapefinders}

For a given surface the four Minkowski functionals (from the first to the fourth) are proportional to the enclosed volume $V$, the area of the surface $S$, the integrated mean curvature $C$, and the integrated Gaussian curvature $\chi$. Consider an excursion set $F_{\phi_{0}}$ of a field $\phi(x)$ (the set of all points where the density is higher than a given limit, $\left.\phi\left(x \geq \phi_{0}\right)\right)$. Then, the first Minkowski functional (the volume functional) is the volume of this region (the excursion set):

$V_{0}\left(\phi_{0}\right)=\int_{F_{\phi_{0}}} \mathrm{~d}^{3} x$.

The second Minkowski functional is proportional to the surface area of the boundary $\delta F_{\phi}$ of the excursion set:

$V_{1}\left(\phi_{0}\right)=\frac{1}{6} \int_{\delta F_{\phi_{0}}} \mathrm{~d} S(\boldsymbol{x})$

(but it is not the area itself, notice the constant). The third Minkowski functional is proportional to the integrated mean curvature $C$ of the boundary:

$V_{2}\left(\phi_{0}\right)=\frac{1}{6 \pi} \int_{\delta F_{\phi_{0}}}\left(\frac{1}{R_{1}(\boldsymbol{x})}+\frac{1}{R_{2}(\boldsymbol{x})}\right) \mathrm{d} S(\boldsymbol{x})$,

where $R_{1}(\boldsymbol{x})$ and $R_{2}(\boldsymbol{x})$ are the principal radii of curvature of the boundary. The fourth Minkowski functional is proportional to the integrated Gaussian curvature (the Euler characteristic) of the boundary:

$V_{3}\left(\phi_{0}\right)=\frac{1}{4 \pi} \int_{\delta F_{\phi_{0}}} \frac{1}{R_{1}(\boldsymbol{x}) R_{2}(\boldsymbol{x})} \mathrm{d} S(\boldsymbol{x})$.
At high (low) densities this functional gives us the number of isolated clumps (void bubbles) in the sample (Martínez \& Saar 2002; Saar et al. 2007):

$V_{3}=N_{\text {clumps }}+N_{\text {bubbles }}-N_{\text {tunnels }}$.

As the argument labelling the isodensity surfaces, we chose the (excluded) mass fraction $m_{f}$ - the ratio of the mass in the regions with the density lower than the density at the surface, to the total mass of the supercluster. When this ratio runs from 0 to 1 , the iso-surfaces move from the outer limiting boundary into the centre of the supercluster, i.e., the fraction $m_{f}=0$ corresponds to the whole supercluster, and $m_{f}=1-$ to its highest density peak.

The first three functionals were used to calculate the shapefinders (Sahni et al. 1998; Shandarin et al. 2004; Saar 2009). The shapefinders are defined as a set of combinations of Minkowski functionals: $H_{1}=3 \mathrm{~V} / \mathrm{S}$ (thickness), $H_{2}=S / C$ (width), and $H_{3}=C / 4 \pi$ (length). The shapefinders have dimensions of length and are normalized to give $H_{i}=R$ for a sphere of radius $R$. For smooth (ellipsoidal) surfaces, the shapefinders $H_{i}$ follow the inequalities $H_{1} \leq H_{2} \leq H_{3}$. Oblate ellipsoids (pancakes) are characterised by $H_{1} \ll H_{2} \approx H_{3}$, while prolate ellipsoids (filaments) are described by $H_{1} \approx H_{2} \ll H_{3}$. Sahni et al. (1998) also defined two dimensionless shapefinders $K_{1}$ (planarity) and $K_{2}$ (filamentarity): $K_{1}=\left(H_{2}-H_{1}\right) /\left(H_{2}+H_{1}\right)$ and $K_{2}=\left(H_{3}-H_{2}\right) /\left(H_{3}+H_{2}\right)$. In the $\left(K_{1}, K_{2}\right)$-plane filaments are located near the $K_{2}$-axis, pancakes near the $K_{1}$-axis, and ribbons along the diameter, connecting the spheres at the origin with the ideal ribbon at $(1,1)$. In Einasto et al. (2007c) we calculated typical morphological signatures of a series of empirical models that serve as morphological templates to compare with the characteristic curves for superclusters in the $\left(K_{1}, K_{2}\right)$-plane. 
Appendix D: Data on selected clusters

Table D.1. Data on most luminous clusters.

\begin{tabular}{|c|c|c|c|c|c|c|c|c|c|c|c|c|c|}
\hline $\begin{array}{l}\text { (1) } \\
\text { ID }\end{array}$ & $\begin{array}{c}(2) \\
N_{\text {gal }}\end{array}$ & $\begin{array}{c}(3) \\
L_{\text {tot }} \\
10^{10} h^{-2} L_{\odot} \\
\end{array}$ & $\begin{array}{c}(4) \\
\sigma \\
\mathrm{km} \mathrm{s}^{-1} \\
\end{array}$ & $\begin{array}{c}(5) \\
r_{\mathrm{vir}} \\
h^{-1} \mathrm{Mpc} \\
\end{array}$ & $\begin{array}{c}(6) \\
V_{\mathrm{pec}} \\
\mathrm{km} \mathrm{s}^{-1} \\
\end{array}$ & $\begin{array}{c}(7) \\
N_{\text {comp }}\end{array}$ & $\begin{array}{l}(8) \\
p_{\Delta}\end{array}$ & $\begin{array}{l}(9) \\
D 4\end{array}$ & $\begin{array}{c}(10) \\
D 8\end{array}$ & $\begin{array}{l}(11) \\
D 16\end{array}$ & $\begin{array}{l}(12) \\
I D_{\mathrm{scl}}\end{array}$ & $\begin{array}{l}\text { (13) } \\
\text { Type }\end{array}$ & $\begin{array}{c}(14) \\
\text { Abell ID }\end{array}$ \\
\hline 914 & 119 & 227 & 657 & 0.83 & -704 & 5 & $<10^{-4}$ & 49.5 & 13.2 & 4.0 & 27 & $\mathrm{~F}$ & A 1750 \\
\hline 29587 & 207 & 365 & 740 & 0.87 & 334 & 3 & $<10^{-4}$ & 79.3 & 21.3 & 5.8 & 1 & $\mathrm{~F}$ & A 2142 \\
\hline 34727 & 256 & 351 & 825 & 1.25 & -290 & 5 & $<10^{-4}$ & 51.3 & 16.1 & 5.1 & 7 & S & A 2028,A 2029,A 2033,A 2040 \\
\hline 38087 & 169 & 209 & 541 & 0.84 & 844 & 2 & 0.031 & 51.1 & 13.9 & 3.7 & 24 & S & A 1173,A 1187,A 1190,A 1203 \\
\hline 39489 & 166 & 188 & 1061 & 0.72 & -521 & 3 & $<10^{-4}$ & 43.5 & 10.7 & 4.0 & 99 & $\mathrm{~S}$ & A 2056, A 2065 \\
\hline 68625 & 92 & 301 & 874 & 0.79 & 825 & 3 & $<10^{-4}$ & 72.6 & 20.0 & 6.3 & 3 & S & A 2069 \\
\hline 73088 & 141 & 184 & 631 & 0.71 & -224 & 3 & $<10^{-4}$ & 47.7 & 11.5 & 3.2 & 92 & $S$ & A 1904 \\
\hline
\end{tabular}

Notes. Columns are as follows: 1 : ID of the cluster; 2 : the number of galaxies in the cluster, $N_{\text {gal }}$; 3 : total luminosity of the cluster; 4 : rms velocity of the cluster; 5: virial radius of the cluster; 6: peculiar velocity of the main galaxy; 7: the number of components in the cluster, $N_{\text {comp }} ; 8: p$-value of the DS test; 9-11: environmental density around the cluster, at smoothing lengths 4, 8, and $16 h^{-1} \mathrm{Mpc}$ (in units of the mean density); 12 : ID of the supercluster where the cluster resides. 13: morphological type of the supercluster 14: Abell ID of the cluster.

Table D.2. Data on unimodal clusters.

\begin{tabular}{|c|c|c|c|c|c|c|c|c|c|c|c|c|c|}
\hline (1) & (2) & (3) & (4) & (5) & (6) & (7) & (8) & (9) & (10) & (11) & (12) & (13) & (14) \\
\hline ID & $N_{\text {gal }}$ & $\begin{array}{c}L_{\mathrm{tot}} \\
10^{10} h^{-2} L_{\odot}\end{array}$ & $\begin{array}{c}\sigma \\
\mathrm{km} \mathrm{s}^{-1}\end{array}$ & $\begin{array}{c}r_{\mathrm{vir}} \\
h^{-1} \mathrm{Mpc}\end{array}$ & $\begin{array}{c}V_{\text {pec }} \\
\mathrm{km} \mathrm{s}^{-1}\end{array}$ & $N_{\text {comp }}$ & $p_{\Delta}$ & $D 4$ & $D 8$ & $D 16$ & $I D_{\mathrm{scl}}$ & Type & Abell ID \\
\hline 608 & 60 & 132 & 532 & 0.66 & -228 & 1 & 0.884 & 33.4 & 7.6 & 2.7 & 569 & $\bar{S}$ & A 2175 \\
\hline 5217 & 89 & 94 & 577 & 0.61 & -19 & 1 & 0.216 & 27.3 & 8.0 & 3.2 & 211 & $\mathrm{~F}$ & A 1436 \\
\hline 25078 & 51 & 87 & 498 & 0.61 & -120 & 1 & 0.270 & 30.0 & 11.8 & 4.9 & 27 & $\mathrm{~F}$ & A 1650 \\
\hline 39914 & 63 & 69 & 446 & 0.65 & 148 & 1 & 0.051 & 27.1 & 8.1 & 3.8 & 99 & $\mathrm{~S}$ & А 2089 \\
\hline 50129 & 52 & 61 & 449 & 0.51 & -129 & 1 & 0.326 & 22.3 & 7.4 & 2.9 & 220 & F & A 1775 \\
\hline 58604 & 58 & 39 & 528 & 0.42 & -201 & 1 & 0.504 & 16.1 & 3.9 & 1.2 & 0 & - & - \\
\hline 67116 & 80 & 114 & 651 & 0.44 & -183 & 1 & 0.094 & 36.2 & 10.5 & 3.8 & 27 & $\mathrm{~F}$ & A 1809 \\
\hline
\end{tabular}

Notes. Columns are as in Table D.1.

Table D.3. Data on multimodal clusters.

\begin{tabular}{|c|c|c|c|c|c|c|c|c|c|c|c|c|c|}
\hline (1) & $(2)$ & (3) & (4) & (5) & (6) & (7) & (8) & (9) & $(10)$ & (11) & $(12)$ & (13) & (14) \\
\hline ID & $N_{\text {gal }}$ & $\begin{array}{c}L_{\text {tot }} \\
10^{10} h^{-2} L_{\odot}\end{array}$ & $\begin{array}{c}\sigma \\
\mathrm{km} \mathrm{s}^{-1}\end{array}$ & $\begin{array}{c}r_{\mathrm{vir}} \\
h^{-1} \mathrm{Mpc}\end{array}$ & $\begin{array}{c}V_{\mathrm{pec}} \\
\mathrm{km} \mathrm{s}^{-1}\end{array}$ & $N_{\text {comp }}$ & $p_{\Delta}$ & $D 4$ & $D 8$ & $D 16$ & $I D_{\mathrm{scl}}$ & Type & Abell ID \\
\hline 880 & 57 & 101 & 411 & 0.84 & 400 & 3 & 0.002 & 26.6 & 6.9 & 2.8 & 27 & $\mathrm{~F}$ & - \\
\hline 4122 & 88 & 68 & 963 & 0.49 & -1091 & 3 & $<10^{-4}$ & 19.8 & 5.6 & 2.7 & 515 & $\mathrm{~S}$ & A 1291 \\
\hline 28387 & 88 & 121 & 481 & 0.66 & -167 & 4 & $<10^{-4}$ & 28.5 & 8.4 & 3.4 & 211 & $\mathrm{~F}$ & - \\
\hline 34726 & 145 & 121 & 506 & 0.74 & -56 & 4 & 0.001 & 32.1 & 8.4 & 2.8 & 352 & $\mathrm{~S}$ & A 2028, A 2033, A 2040 \\
\hline 58305 & 167 & 120 & 401 & 0.61 & 115 & 3 & $<10^{-4}$ & 30.2 & 6.9 & 2.4 & 541 & S & A 1983 \\
\hline 67297 & 95 & 86 & 770 & 0.45 & 123 & 2 & 0.001 & 26.5 & 5.4 & 1.5 & 1104 & $\mathrm{~S}$ & А 671 \\
\hline 68625 & 92 & 301 & 874 & 0.79 & 825 & 3 & $<10^{-4}$ & 72.6 & 20.0 & 6.3 & 3 & S & A 2069 \\
\hline 73088 & 141 & 184 & 631 & 0.71 & -224 & 3 & $<10^{-4}$ & 47.7 & 11.5 & 3.2 & 92 & S & A 1904 \\
\hline
\end{tabular}

Notes. Columns are as in Table D.1. 